\title{
Phase diagrams of interacting spreading dynamics in complex networks
}

\author{
Liming Pan $\odot,{ }^{1,2}$ Dan Yang $\odot,{ }^{2}$ Wei Wang $\odot,{ }^{3,2, *}$ Shimin Cai, ${ }^{2,4,5}$ Tao Zhou, ${ }^{2,4,5}$ and Ying-Cheng Lai $\odot^{6}$ \\ ${ }^{1}$ School of Computer Science and Technology, Nanjing Normal University, Nanjing, \\ Jiangsu 210023, China \\ ${ }^{2}$ Complex Lab, School of Computer Science and Engineering, University of Electronic Science and Technology of China, \\ Chengdu 611731, China \\ ${ }^{3}$ Cybersecurity Research Institute, Sichuan University, Chengdu 610065, China \\ ${ }^{4}$ Institute of Fundamental and Frontier Sciences, University of Electronic Science and Technology of China, Chengdu 610054, China \\ ${ }^{5}$ Big Data Research Center, University of Electronic Science and Technology of China, Chengdu 610054, China \\ ${ }^{6}$ School of Electrical, Computer and Energy Engineering, Arizona State University, Tempe, Arizona 85287, USA
}

(Received 29 August 2019; revised manuscript received 24 November 2019; accepted 7 May 2020; published 27 May 2020)

\begin{abstract}
Epidemic spreading processes in the real world can interact with each other in a cooperative, competitive, or asymmetric way, requiring a description based on coevolution dynamics. Rich phenomena such as discontinuous outbreak transitions and hystereses can arise, but a full picture of these behaviors in the parameter space is lacking. We develop a theory for interacting spreading dynamics on complex networks through spectral dimension reduction. In particular, we derive from the microscopic quenched mean-field equations a twodimensional system in terms of the macroscopic variables, which enables a full phase diagram to be determined analytically. The diagram predicts critical phenomena that were known previously but only numerically, such as the interplay between discontinuous transition and hysteresis as well as the emergence and role of tricritical points.
\end{abstract}

DOI: 10.1103/PhysRevResearch.2.023233

\section{INTRODUCTION}

Spreading dynamics of diseases, behaviors, and information in nature and human society are rarely independent processes but interact with each other in a complex way. Weakened immunity to other viruses due to HIV infection $[1,2]$ and suppression of spreading due to disease-related information exchange on the social media [3] are known examples. To better understand, predict, and control spreading on networks, coevolution of epidemics must be taken into account. In network science, there has been continuous interest in developing interacting epidemic models [1,2,4-12], which can generate surprising behaviors that cannot be predicted by any single-virus epidemic model. For example, spreading of one epidemic can facilitate that of another, leading to a firstorder or explosive transition in the outbreak with significant real-world implications [13]. Many factors can affect the critical behaviors of interacting spreading dynamics, such as self-evolution of each epidemic [3,14], interaction between two epidemics [15,16], and network structure [17,18].

By now, spreading dynamics of a single epidemic on complex networks have been well studied [8,19-21].

\footnotetext{
*wwzqbx@hotmail.com

Published by the American Physical Society under the terms of the Creative Commons Attribution 4.0 International license. Further distribution of this work must maintain attribution to the author(s) and the published article's title, journal citation, and DOI.
}

For interacting spreading dynamics, the special case of well-mixed populations has been treated [22,23]. A study based on the quenched mean field for two competing pathogens [24] showed that, when simultaneous infection by the two pathogens is not possible (full mutual exclusion), the phase diagram is independent of the spectral radius of the network. There were also theories based on percolation [25], annealed mean field [26], and pair approximations [17] to study the effect of network structure on interacting spreading, leading to a qualitative understanding of critical phenomena. There are difficulties with these theories. For example, the annealed mean-field theory takes into account only the nodal degrees and is not applicable to quenched networks (especially networks with a high clustering coefficient and modularity). For such networks, quenched mean-field theories [27] such as those based on Markov chains [28] and the $N$-intertwined method [29] is needed. A deficiency of such a theory is that it uses a large number of nonlinear differential equations, with two difficulties: (a) high computational overload for large networks and (b) lack of any analytic insights. Such a theory, due to its heavy reliance on numerics, can lead to inconsistent or even contradicting predictions [30,31]. To our knowledge, a general analytic theory capable of providing a more complete understanding of interacting spreading dynamics is lacking.

In this paper, we develop an analytic theory for interacting spreading dynamics on complex networks through the approach of dimension reduction for complex networks [32-34]. From the quenched mean-field equations, we derive a two-dimensional (2D) system that is capable of analytically yielding the full phase diagrams underlying interacting 
spreading dynamics on any complex network, from which the conditions for various phase transitions can be derived. The analytic model predicts critical phenomena that were previously known numerically, such as the interplay between discontinuous outbreak transitions and hystereses as well as the emergence of tricritical points, providing a solid theoretical foundation for understanding interacting spreading dynamics and articulating optimal control strategies.

\section{MODEL, METHOD OF SPECTRAL DIMENSION REDUCTION, AND REDUCED MODEL}

\section{A. Model}

We consider the susceptible-infected-susceptible (SIS) model of interacting spreading dynamics on complex networks. In the classic SIS model, a single epidemic spreads in the network and a node can be either in the susceptible or in the infected state. Susceptible nodes are infected by their infected neighbors at rate $\lambda$ and infected nodes recover at rate $\gamma$. For interacting SIS dynamics, two epidemics, say 1 and 2, spread simultaneously and interact with each other. Each node infected by $a \in\{1,2\}$ transmits the infection to neighbors that are susceptible for both epidemics with probability $\lambda_{a}$. If a neighbor is susceptible for $a$ but infected by the other epidemic, then the infection will be transmitted with rate $\lambda_{a}^{\dagger}$. All the nodes infected by $a$ recover to being susceptible with rate $\gamma_{a}$. Without loss of generality, we set $\gamma_{a}=1$ for both $a \in$ $\{1,2\}$. In general, the nature of the interacting SIS dynamics depends on the interplay between the rates $\lambda_{a}$ and $\lambda_{a}^{\dagger}$. In particular, for $\lambda_{a}^{\dagger}>\lambda_{a}$, the two epidemics tend to facilitate each other, leading to cooperative SIS dynamics, whereas if $\lambda_{a}^{\dagger}<\lambda_{a}$, infection with one epidemic will suppress infection with the other, giving rise to competitive SIS dynamics. For $\lambda_{a}^{\dagger}>\lambda_{a}$ but $\lambda_{b}^{\dagger}<\lambda_{b}$ for $a, b \in\{1,2\}$ with $b \neq a$, the interactions are asymmetric.

\section{B. Spectral dimension reduction}

The interacting SIS model represents a paradigm to study rich dynamical behaviors such as first-order outbreak transitions and hystereses [35]. The foundation of our study of this model is the quenched mean-field theory (QMF) [36]. While in QMF the dynamical correlations among the neighbors are assumed to be negligible, the theory has been demonstrated to generate reliable prediction of the phase transitions [37]. Since our goal is to analytically map out the complete phase diagram, using the QMF suffices. Let $p_{a, i}$ be the probability that node $i \in\{1, \cdots, N\}$ is infected by $a \in\{1,2\}$ at time $t$. In the first-order mean-field analysis [27], the evolution of $p_{a, i}$ on a network with adjacency matrix $G$ is governed by

$$
\begin{aligned}
\frac{d p_{a, i}}{d t}= & -p_{a, i}+\lambda_{a}^{\dagger}\left(1-p_{a, i}\right) p_{b, i} \sum_{j} G_{i j} p_{a, j} \\
& +\lambda_{a}\left(1-p_{a, i}\right)\left(1-p_{b, i}\right) \sum_{j} G_{i j} p_{a, j}
\end{aligned}
$$

for $a \in\{1,2\}$ and $i \in\{1, \cdots, N\}$. The first term on the right side of Eq. (1) is the rate of recovery from epidemic $a$ for node $i$, while the second (third) term corresponds to the rate of infection for epidemic $a$ with (without) $i$ already infected by $b \neq a$. For a network of size $N$, the number of equations in Eq. (1) is $2 N$. To derive an analytic model, we exploit the technique of spectral dimension reduction (SDR) [33] to arrive at an equivalent description of the original system in terms of two macroscopic observables-one for each epidemic. In particular, let $\alpha$ be a vector with nonnegative entries and normalized as $\sum_{i} \alpha_{i}=1$. The entries of $\alpha$ represent the nodal weights. We define linear observables as $\psi_{a}=\alpha^{T} p_{a}$ for $a \in\{1,2\}$. Since the entries of $\alpha$ are summed to unity, $\psi_{a}$ is a weighted average. The evolution of $\psi_{a}$ is determined by the equation

$$
\frac{d \psi_{a}}{d t}=\sum_{i=1}^{N} \alpha_{i} \frac{d p_{a, i}}{d t} .
$$

Applying the SDR method, we have that the right-hand side of Eq. (2) can be written in terms of the macroscopic observables $\psi_{a}$ only.

\section{Reduced model}

We apply the SDR method to Eq. (2). Microscopic variables $p_{a, i}$ fluctuate about the macroscopic observables $\psi_{a}$, which can be decomposed as

$$
\begin{aligned}
p_{a, i} & =\rho_{a} \psi_{a}+\delta p_{a, i}, \\
p_{b, i} & =\mu_{a} \psi_{b}+\delta p_{b, i}, \\
p_{a, j} & =v_{a} \psi_{a}+\delta p_{a, j},
\end{aligned}
$$

where $\rho_{a}, \mu_{a}$, and $\nu_{a}$ are parameters to be determined, and $\delta p_{a, i}, \delta p_{b, i}$, and $\delta p_{a, j}$ are correction terms. Substituting Eqs. (1) and (3) into Eq. (2) gives

$$
\begin{aligned}
\frac{d \psi_{a}}{d t}= & -\psi_{a}+\lambda_{a}^{\dagger} \hat{\alpha} \mu_{a} v_{a}\left(1-\rho_{a} \psi_{a}\right) \psi_{b} \psi_{a} \\
& +\lambda_{a} \hat{\alpha} v_{a}\left(1-\rho_{a} \psi_{a}\right)\left(1-\mu_{a} \psi_{b}\right) \psi_{a}+R_{a},
\end{aligned}
$$

where $\hat{\alpha}=\sum_{i, j} \alpha_{i} G_{i j}$ and $R_{a}$ is the remainder term that can be decomposed as

$$
R_{a}=R_{a, 1}+R_{a, 2}+R_{a, 3},
$$

with $R_{a, 1}, R_{a, 2}$, and $R_{a, 3}$ containing the first-, second-, and third-order terms in the corrections $\left\{\delta p_{a, i}\right\}$, respectively. Let $K$ be the diagonal matrix with $K_{i i}$ being the degree of node $i$, the first-order correction $R_{a, 1}$ is given by

$$
\begin{aligned}
R_{a, 1}= & {\left[\left(\lambda_{a}-\lambda_{a}^{\dagger}\right) \mu_{a} v_{a} \psi_{b} \psi_{a}-\lambda_{a} v_{a} \psi_{a}\right] \alpha^{T} K \delta p_{a} } \\
& +\left(\lambda_{a}^{\dagger}-\lambda_{a}\right) v_{a}\left(1-\rho_{a} \psi_{a}\right) \psi_{a} \alpha^{T} K \delta p_{b} \\
& +\left(1-\rho_{a} \psi_{a}\right)\left(\lambda_{a}^{\dagger} \mu_{a} \psi_{b}+\lambda_{a}-\lambda_{a} \mu_{a} \psi_{b}\right) \alpha^{T} G \delta p_{a},
\end{aligned}
$$

where $\delta p_{a}$ is a vector with $\delta p_{a, i}$ in the $i$ th entry and $\delta p_{b}$ is defined analogously. The second-order remainder term is

$$
\begin{aligned}
R_{a, 2}= & \left(\lambda_{a}^{\dagger}-\lambda_{a}\right)\left(1-\rho_{a} \psi_{a}\right) \sum_{i=1}^{N} \sum_{j=1}^{N} G_{i j} \alpha_{i} \delta p_{b, i} \delta p_{a, j} \\
& +\left[\left(\lambda_{a}-\lambda_{a}^{\dagger}\right) \mu_{a} \psi_{b}-\lambda_{a}\right] \sum_{i=1}^{N} \sum_{j=1}^{N} G_{i j} \alpha_{i} \delta p_{a, i} \delta p_{a, j} \\
& +\left(\lambda_{a}-\lambda_{a}^{\dagger}\right) \nu_{a} \psi_{a} \sum_{i=1}^{N} \sum_{j=1}^{N} G_{i j} \alpha_{i} \delta p_{a, i} \delta p_{b, i}
\end{aligned}
$$


and the third-order remainder term is

$$
R_{a, 3}=\left(\lambda_{a}-\lambda_{a}^{\dagger}\right) \sum_{i=1}^{N} \sum_{j=1}^{N} G_{i j} \alpha_{i} \delta p_{a, i} \delta p_{b, i} \delta p_{a, j} .
$$

From Eq. (6), the dominant remainder term $R_{a, 1}$ vanishes if the following equations hold

$$
\begin{aligned}
\alpha^{T} K p_{a} & =\hat{\alpha} \rho_{a} \psi_{a}, \\
\alpha^{T} K p_{b} & =\hat{\alpha} \mu_{a} \psi_{b}, \\
\alpha^{T} G p_{a} & =\hat{\alpha} v_{a} \psi_{a},
\end{aligned}
$$

where $p_{a}$ is a vector with $p_{a, i}$ in the $i$ th entry and $p_{b}$ is defined similarly.

In general, the equations cannot be satisfied simultaneously. An application of the SDR method in Ref. [33] advocates choosing $\alpha$ as the eigenvector associated with the leading eigenvalue $\omega$ of $G$. For connected undirected networks, the eigenvector associated with the leading eigenvalue $\omega$ of $G$ has positive entries. The third equation in Eqs. (9) implies $\omega \psi_{a}=\hat{\alpha} v_{a} \psi_{a}$ and, hence, $\omega=v_{a} \hat{\alpha}$. Using the definition $\hat{\alpha}=$ $\mathbf{1}^{T} G \alpha=\omega$, we have $v_{a}=1$. The remaining two parameters, $\rho_{a}$ and $\mu_{b}$, are chosen such that the first two equations in Eqs. (9) are satisfied. The quantities $\rho_{a}$ and $\mu_{b}$ can be chosen by minimizing the following squared vector norm

$$
\rho_{a}^{*}=\mu_{a}^{*}=\underset{x}{\operatorname{argmin}}\|K \alpha-x \hat{\alpha} \alpha\|_{2}^{2},
$$

which yields

$$
\mu:=\frac{1}{\omega} \frac{\alpha^{T} K \alpha}{\alpha^{T} \alpha}=\rho_{a}^{*}=\mu_{a}^{*} .
$$

A justification of the parameter choices was given in Ref. [33]. With the parameters chosen, $R_{a, 1}$ can be made as small as possible and can be neglected, so can the higher-order terms $R_{a, 2}$ and $R_{a, 3}$. Substituting the values of $\rho_{a}, \mu_{a}$, and $v_{a}$ into Eq. (4), we get

$$
\begin{aligned}
\frac{d \psi_{a}}{d t}= & -\psi_{a}+\lambda_{a}^{\dagger} \omega \mu\left(1-\mu \psi_{a}\right) \psi_{b} \psi_{a} \\
& +\lambda_{a} \omega\left(1-\mu \psi_{a}\right)\left(1-\mu \psi_{b}\right) \psi_{a}+R_{a} .
\end{aligned}
$$

The first term on the right side of Eq. (11) accounts for the rate of recovery and the second (third) term represents the rate of infection for epidemic $a$ with (without) being infected by $b \neq a$. The quantity $R_{a}$ in Eq. (11) characterizes the fluctuations of the microscopic observables $p_{a, i}$ about the macroscopic observables $\psi_{a}$, which is small in comparison to other terms on the right side of Eq. (11) due to $\alpha$ 's being the leading eigenvector. Since, for finding the phase diagram, it is necessary to analyze the mean-field equations that depend on the macroscopic observables $\psi_{a}$ only, it is justified to drop $R_{a}$ from the analysis. As we will verify numerically, this approximation will not affect the accuracy of the phase diagram as the resulting errors near the phase boundaries are quite insignificant.

In Eq. (1), the order parameters are $\left\langle p_{a, i}\right\rangle$ for $a \in\{1,2\}$, where $\langle\cdot\rangle$ is the unweighted average over the nodes. In Eq. (11), the order parameters can be chosen to be $\psi_{a}=\alpha^{T} p_{a}$, a weighted average over the nodes. Since $\alpha$ is the eigenvector associated with the leading eigenvalue of $G$, its entries are strictly positive. As a result, $\psi_{a}=0\left(\psi_{a}>0\right)$ implies $\left\langle p_{a, i}\right\rangle=0\left(\left\langle p_{a, i}\right\rangle>0\right)$. When crossing a phase boundary, at least one of $\psi_{a}$ for $a \in\{1,2\}$ becomes either zero or nonzero, guaranteeing that the corresponding $\left\langle p_{a, i}\right\rangle$ becomes either zero or nonzero, respectively. We have that $\left\langle p_{a, i}\right\rangle$ and $\psi_{a}$ give the same phase diagram, which can be obtained analytically through the 2D mean-field system.

\section{MAIN RESULT: PHASE DIAGRAM OF REDUCED SYSTEM}

The reduced mean-field equations are amenable to analytic treatment. As the derivations are lengthy, we provide a brief sketch of the results from analyzing the reduced system.

The analyses of the 2D mean-field system are performed in the following steps. First, for each point in the parameter space $\left(\lambda_{1}, \lambda_{1}^{\dagger}, \lambda_{2}, \lambda_{2}^{\dagger}\right)$, we determine the equilibrium points of Eq. (11) (Sec. III A) and their stability (Sec. III B). The the equilibrium points have to further satisfy the probability constraint $1 \leqslant \psi_{a} \leqslant 1$ to be physical meaningful. A detailed analysis of the stability and probability constraints of the equilibrium points leads to the following functions of $\lambda_{a}$ and $\lambda_{a}^{\dagger}$ :

$$
\begin{aligned}
s_{a, 0}= & \lambda_{b}+\lambda_{a}^{\dagger}-\lambda_{a}-\omega \lambda_{b} \lambda_{a}^{\dagger}, \\
s_{a, 1}= & \lambda_{b}^{\dagger}-\lambda_{b}-\lambda_{a}^{\dagger}+\lambda_{a}+2 \omega \lambda_{b} \lambda_{a}^{\dagger}-\omega \lambda_{a}^{\dagger} \lambda_{b}^{\dagger}, \\
s_{a, 2}= & \lambda_{b}^{\dagger}-\lambda_{b}, s_{a, 3}=\lambda_{a}-\omega^{-1}, \\
s_{\Delta}= & \left(\lambda_{1}^{\dagger}-\lambda_{1}+\lambda_{2}^{\dagger}-\lambda_{2}-\omega \lambda_{1}^{\dagger} \lambda_{2}^{\dagger}\right)^{2}, \\
& -4\left(\lambda_{1}^{\dagger}-\lambda_{1}\right)\left(\lambda_{2}^{\dagger}-\lambda_{2}\right) .
\end{aligned}
$$

for $a, b \in\{1,2\}$ and $a \neq b$. Whether an equilibrium point is physical or stable is determined by the signs of these functions.

Calculating the equilibrium points (Sec. III A) and analyzing their stability (Sec. III B) enable us to obtain the full phase diagram of the reduced system and the equations for the phase boundaries (Sec. III C). Based on the numbers of stable and unstable equilibrium points as well as the relationships among them, we can divide the parameter space into distinct regions, where a boundary crossing between two neighboring regions gives rise to a phase transition. A region either can have a unique stable equilibrium point or can have two stable equilibrium points with one unstable point in between, where crossing the latter will result in a hysteresis. We discuss the types of phase transitions crossing the various boundaries and study the interplay between the transitions and the phenomenon of hysteresis (Sec. IIID). Finally, we derive the conditions under which a hysteresis can arise (Sec. IIIE).

The analytical results of the full phase diagram are summarized and discussed in Sec. III F. Readers who are not interested in the technical details of analyzing the 2D mean-field system can skip Secs. III A to III E and check Sec. III F for the results. For convenience, for the rest of the paper, we use the convention that, if variables indexed by $a, b \in\{1,2\}$ (e.g., $\psi_{a}$ and $\psi_{b}$ ) appear together in an equation or an inequality, then the assumption is $a \neq b$. 


\section{A. Equilibrium points of the reduced system}

The equilibrium points are obtained by setting the right side of Eq. (11) to zero:

$$
\begin{aligned}
& -\psi_{a}+\lambda_{a}^{\dagger} \omega \mu\left(1-\mu \psi_{a}\right) \psi_{b} \psi_{a} \\
& +\lambda_{a} \omega\left(1-\mu \psi_{a}\right)\left(1-\mu \psi_{b}\right) \psi_{a}=0
\end{aligned}
$$

for $a, b \in\{1,2\}$ and $a \neq b$. Further, the physical solutions have to satisfy the probability constraints $0 \leqslant \psi_{a} \leqslant 1$. Because of the appearance of terms such as $\left(1-\mu \psi_{a}\right)$ in Eq. (11), it is necessary to impose the physical condition $\left(1-\mu \psi_{a}\right) \leqslant 1$. It can be proved that $\mu$ given by Eq. (10) satisfies $\mu \geqslant 1$ (see the Appendix for a proof), and it can also be verified that any point with $\psi_{1}=\mu^{-1}$ or $\psi_{2}=\mu^{-1}$ cannot be an equilibrium point. These, together the probability constraints, imply that all the equilibrium points must satisfy the inequality $0 \leqslant \psi_{a}<\mu^{-1}$ for $a \in\{1,2\}$.

We are now in a position to discuss the types of equilibrium points of the reduced mean-field equations.

(i) Epidemic free. The trivial solution $\left(\psi_{1}, \psi_{2}\right)=(0,0)$ is always an equilibrium point.

(ii) Partial infection of epidemic 1 . For $\psi_{1} \neq 0$ and $\psi_{2}=0$, Eqs. (13) become

$$
-1+\lambda_{1} \omega\left(1-\mu \psi_{1}\right)=0,
$$

which gives

$$
\psi_{1}=\frac{\lambda_{1} \omega-1}{\mu \lambda_{1} \omega} .
$$

The solution further has to satisfy the probability constraint $0<\psi_{1} \leqslant \mu^{-1}$. The first inequality $\psi_{1}>0$ is satisfied when $\lambda_{1} \omega>1$, while the second inequality $\psi_{1} \leqslant \mu^{-1}$ always holds.

(iii) Partial infection of epidemic 2. Similar to case (ii), we have the equilibrium point:

$$
\left(\psi_{1}, \psi_{2}\right)=\left(0, \frac{\lambda_{2} \omega-1}{\mu \lambda_{2} \omega}\right) .
$$

(iv) Coexistence. If $\psi_{a} \neq 0$ for both $a \in\{1,2\}$, then Eqs. (13) become

$$
\begin{aligned}
& \lambda_{1}^{\dagger} \omega \mu\left(1-\mu \psi_{1}\right) \psi_{2}+\lambda_{1} \omega\left(1-\mu \psi_{1}\right)\left(1-\mu \psi_{2}\right)=1, \\
& \lambda_{2}^{\dagger} \omega \mu\left(1-\mu \psi_{2}\right) \psi_{1}+\lambda_{2} \omega\left(1-\mu \psi_{2}\right)\left(1-\mu \psi_{1}\right)=1 .
\end{aligned}
$$

Rearranging the second equation, we get

$$
\psi_{2}=\mu^{-1}-\frac{1}{\left(\lambda_{2}^{\dagger}-\lambda_{2}\right) \mu^{2} \omega \psi_{1}+\lambda_{2} \mu \omega} .
$$

Substituting this relation into Eq. (17), we get an equation that depends on $\psi_{1}$ only. Similarly we can obtain the equation that determines $\psi_{2}$. The two equations for $\psi_{1}$ and $\psi_{2}$ have the following symmetric form:

$$
g_{a, 2} \psi_{a}^{2}+g_{a, 1} \psi_{a}+g_{a, 0}=0
$$

for $a \in\{1,2\}$, where

$$
\begin{aligned}
& g_{a, 2}=\omega^{2} \mu^{3} \lambda_{a}^{\dagger}\left(\lambda_{b}^{\dagger}-\lambda_{b}\right), \\
& g_{a, 1}=\omega \mu^{2}\left(\lambda_{b}^{\dagger}-\lambda_{b}-\lambda_{a}^{\dagger}+\lambda_{a}+2 \omega \lambda_{b} \lambda_{a}^{\dagger}-\omega \lambda_{a}^{\dagger} \lambda_{b}^{\dagger}\right), \\
& g_{a, 0}=\omega \mu\left(\lambda_{b}+\lambda_{a}^{\dagger}-\lambda_{a}-\omega \lambda_{b} \lambda_{a}^{\dagger}\right)
\end{aligned}
$$

for $b \in\{1,2\}$ and $b \neq a$.
If $\lambda_{a}^{\dagger} \neq \lambda_{a}$ holds for $a \in\{1,2\}$, then $g_{a, 2} \neq 0$ and Eq. (19) will be quadratic, leading to two solutions,

$$
\psi_{a}^{ \pm}=\frac{-g_{a, 1} \pm \sqrt{g_{a, 1}^{2}-4 g_{a, 2} g_{a, 0}}}{2 g_{a, 2}} .
$$

The solutions for $a \in\{1,2\}$ are paired as

$$
\left(\psi_{1}, \psi_{2}\right)=\left(\psi_{1}^{+}, \psi_{2}^{+}\right),\left(\psi_{1}, \psi_{2}\right)=\left(\psi_{1}^{-}, \psi_{2}^{-}\right) .
$$

If $\lambda_{a}^{\dagger}=\lambda_{a}$ for one or both $a \in\{1,2\}$, then we have

$$
\psi_{a}=-\frac{g_{a, 0}}{g_{a, 1}} .
$$

To discuss the probability constraints of the equilibrium points, we consider the following cases.

(iv.1) If $g_{a, 2}=0$ for one of $a \in\{1,2\}$, i.e., $\lambda_{b}^{\dagger}=\lambda_{b}$, then there is a unique solution given by

$$
\psi_{a}=\mu^{-1}-\frac{\lambda_{b}}{\mu\left(\lambda_{a}-\lambda_{a}^{\dagger}+\omega \lambda_{b} \lambda_{a}^{\dagger}\right)}, \quad \psi_{b}=\mu^{-1}-\frac{1}{\mu \omega \lambda_{b}} .
$$

The probability constraints imply

$$
\lambda_{a}-\lambda_{b}-\lambda_{a}^{\dagger}+\omega \lambda_{b} \lambda_{a}^{\dagger}>0, \omega \lambda_{b}>1 .
$$

Further, if we have $\lambda_{a}^{\dagger}=\lambda_{a}$, then the two epidemics will become independent of each other with the solution

$$
\psi_{a}=\mu^{-1}-\frac{1}{\omega \mu \lambda_{a}}, \psi_{b}=\mu^{-1}-\frac{1}{\omega \mu \lambda_{b}} .
$$

(iv.2) Suppose $\lambda_{a}^{\dagger}>\lambda_{a}$ for both $a \in\{1,2\}$. In this case there are two solutions, as shown in Eqs. (22).

Consider the solution

$$
\left(\psi_{1}, \psi_{2}\right)=\left(\psi_{1}^{+}, \psi_{2}^{+}\right) .
$$

First, it is necessary to have $g_{a, 1}^{2}-4 g_{a, 2} g_{a, 0} \geqslant 0$ for $a \in\{1,2\}$ to make the solutions real. Because of the condition $g_{a, 2}>0$, the probability constraints $0<\psi_{a}<\mu^{-1}$ imply

$$
g_{a, 1}<\sqrt{g_{a, 1}^{2}-4 g_{a, 2} g_{a, 0}}<2 \mu^{-1} g_{a, 2}+g_{a, 1}
$$

for $a \in\{1,2\}$. The second inequality can be written as

$$
\mu^{-2} g_{a, 2}+\mu^{-1} g_{a, 1}+g_{a, 0}>0 .
$$

Substituting these into Eqs. (20), we have

$$
g_{a, 0}+\mu^{-1} g_{a, 1}+\mu^{-2} g_{a, 2}=\mu \omega \lambda_{b}^{\dagger}>0,
$$

indicating that the second inequality always holds.

It remains to consider the first inequality in Eq. (27). Suppose $g_{a, 0}<0$, then both the first and the inequality $g_{a, 1}^{2}-$ $4 g_{a, 2} g_{a, 0} \geqslant 0$ hold. Otherwise, suppose $g_{a, 0}>0$, it is necessary to have $g_{a, 1}<0$ and $g_{a, 1}^{2}-4 g_{a, 2} g_{a, 0} \geqslant 0$.

Combining the discussions above, we have that $\left(\psi_{1}^{+}, \psi_{2}^{+}\right)$ is a physical solution either for $g_{a, 0}<0$ or for $g_{a, 0}>0, g_{a, 1}<$ $0, g_{a, 1}^{2}-4 g_{a, 2} g_{a, 0} \geqslant 0$ for both $a \in\{1,2\}$.

We now consider the solution $\left(\psi_{1}, \psi_{2}\right)=\left(\psi_{1}^{-}, \psi_{2}^{-}\right)$. For the solution to be meaningful, it has to be guaranteed that $g_{a, 1}^{2}-4 g_{a, 2} g_{a, 0} \geqslant 0$ for $a \in\{1,2\}$. The probability constraints give

$$
g_{a, 1}<-\sqrt{g_{a, 1}^{2}-4 g_{a, 2} g_{a, 0}}<2 \mu^{-1} g_{a, 2}+g_{a, 1} .
$$


The first inequality implies $g_{a, 1}<0$ and $g_{a, 0}>0$. Consider the second inequality. If $2 \mu^{-1} g_{a, 2}+g_{a, 1}>0$, then the second inequality will be satisfied. Else, if $2 \mu^{-1} g_{a, 2}+g_{a, 1} \leqslant 0$, then the second inequality can be written as

$$
\mu^{-2} g_{a, 2}+\mu^{-1} g_{a, 1}+g_{a, 0} \leqslant 0 .
$$

Substituting these into Eqs. (20) we have

$$
g_{a, 0}+\mu^{-1} g_{a, 1}+\mu^{-2} g_{a, 2}=\mu \omega \lambda_{b}^{\dagger}>0,
$$

which leads to a contradiction. It is thus necessary to have $2 \mu^{-1} g_{a, 2}+g_{a, 1} \geqslant 0$ for $a \in\{1,2\}$. In fact, the inequalities $g_{a, 1}<0$ and $g_{a, 0}>0$ are sufficient to guarantee the condition $2 \mu^{-1} g_{a, 2}+g_{a, 1} \geqslant 0$. For $g_{1,1}+g_{2,1}<0$, we have

$$
\lambda_{1}^{\dagger}+\lambda_{2}^{\dagger}-\omega \lambda_{1}^{\dagger} \lambda_{2}^{\dagger}<0 \text {. }
$$

We then have

$$
\begin{aligned}
2 \mu^{-1} g_{1,2}+g_{1,1} & =\omega \mu^{2}\left(\lambda_{2}^{\dagger}-\lambda_{2}-\lambda_{1}^{\dagger}+\lambda_{1}+\omega \lambda_{1}^{\dagger} \lambda_{2}^{\dagger}\right) \\
& >\omega \mu^{2}\left(2 \lambda_{2}^{\dagger}-\lambda_{2}+\lambda_{1}\right)>0 .
\end{aligned}
$$

Similarly, we obtain $2 \mu^{-1} g_{2,2}+g_{2,1}>0$.

Combining the conditions discussed above, we have that the solution $\left(\psi_{1}, \psi_{2}\right)=\left(\psi_{1}^{-}, \psi_{2}^{-}\right)$is physical for $g_{a, 2}>0$, $g_{a, 1}<0, g_{a, 0}>0$, and $g_{a, 1}^{2}-4 g_{a, 2} g_{a, 0}>0$ for $a \in\{1,2\}$. Comparing with the conditions for $\left(\psi_{1}, \psi_{2}\right)=\left(\psi_{1}^{+}, \psi_{2}^{+}\right)$, we see that, for $g_{a, 0}<0$, only one physical solution is possible.

(iv.3) Suppose $\lambda_{a}^{\dagger}<\lambda_{a}$ for $a \in\{1,2\}$ and $\lambda_{b}^{\dagger}>\lambda_{b}$ for $b \in$ $\{1,2\}$ and $b \neq a$. We have $g_{a, 2}>0$ and $g_{b, 2}<0$. Consider the solution $\left(\psi_{1}, \psi_{2}\right)=\left(\psi_{1}^{+}, \psi_{2}^{+}\right)$. The probability constraints imply

$$
\begin{aligned}
& g_{a, 1}<\sqrt{g_{a, 1}^{2}-4 g_{a, 2} g_{a, 0}}<2 \mu^{-1} g_{a, 2}+g_{a, 1}, \\
& g_{b, 1}>\sqrt{g_{b, 1}^{2}-4 g_{b, 2} g_{b, 0}}>2 \mu^{-1} g_{b, 2}+g_{b, 1} .
\end{aligned}
$$

From Eq. (35) we must have either $g_{a, 0}<0$ or $g_{a, 0}>$ $0, g_{a, 1}<0, g_{a, 1}^{2}-4 g_{a, 2} g_{a, 0}>0$. The first inequality in Eq. (35b) implies $g_{b, 1}>0$ and $g_{b, 0}<0$. Now consider the second inequality in Eq. (35b). For $2 \mu^{-1} g_{b, 2}+g_{b, 1} \leqslant 0$, the second inequality in Eq. (35b) holds. Otherwise, if $2 \mu^{-1} g_{b, 2}+$ $g_{b, 1}>0$, then the second inequality implies

$$
c_{b, 0}+\mu^{-1} c_{b, 1}+\mu^{-2} c_{b, 2}>0
$$

which always holds since the left side of the above inequality equals $\mu \omega \lambda_{a}^{\dagger}$.

Recall that, from Eq. (35), we can have either $g_{a, 0}<0$ or $g_{a, 0}>0, g_{a, 1}<0, g_{a, 1}^{2}-4 g_{a, 2} g_{a, 0}>0$. We can show that the latter case contradicts with the conditions $g_{b, 1}>0$ and $g_{b, 0}<$ 0 . In particular, from

$$
g_{b, 0}=\omega \mu\left[\lambda_{a}\left(1-\omega \lambda_{b}^{\dagger}\right)+\lambda_{b}^{\dagger}-\lambda_{b}\right]<0,
$$

we have $1-\omega \lambda_{b}^{\dagger}<0$ and similarly

$$
g_{a, 0}=\omega \mu\left[\lambda_{b}\left(1-\omega \lambda_{a}^{\dagger}\right)+\lambda_{a}^{\dagger}-\lambda_{a}\right]>0,
$$

implying $1-\omega \lambda_{a}^{\dagger}>0$. Since

$$
\begin{aligned}
g_{a, 1} & =\omega \mu^{2}\left(\lambda_{b}^{\dagger}-\lambda_{b}-\lambda_{a}^{\dagger}+\lambda_{a}+2 \omega \lambda_{b} \lambda_{a}^{\dagger}-\omega \lambda_{a}^{\dagger} \lambda_{b}^{\dagger}\right) \\
& =\omega \mu^{2}\left[\left(\lambda_{b}^{\dagger}-\lambda_{b}\right)\left(1-\omega \lambda_{a}^{\dagger}\right)+\lambda_{a}-\lambda_{a}^{\dagger}\left(1-\omega \lambda_{b}\right)\right],
\end{aligned}
$$

then $g_{a, 1}<0$ implies

$$
\lambda_{a}-\lambda_{a}^{\dagger}\left(1-\omega \lambda_{b}\right)<0 .
$$

As a result, we have $\left(1-\omega \lambda_{b}\right)>\lambda_{a} / \lambda_{a}^{\dagger}>1$ and $\omega \lambda_{b}<0$, leading to a contradiction.

Summarizing the above discussions about the equilibrium points, we have that $\left(\psi_{1}, \psi_{2}\right)=\left(\psi_{1}^{+}, \psi_{2}^{+}\right)$is physical for $g_{a, 0}<0, g_{b, 0}<0$ and $g_{b, 1}>0$. Note that $g_{b, 1}>0$ is implied by the other two. Since

$$
g_{b, 1}+\mu g_{b, 0}=\omega \mu^{2}\left[\lambda_{a}^{\dagger}+\omega \lambda_{b}^{\dagger}\left(\lambda_{a}-\lambda_{a}^{\dagger}\right)\right]>0,
$$

we have that $g_{b, 1}>0$ always holds given $g_{b, 0}<0$. Together, it is sufficient to have $g_{a, 0}<0$ and $g_{b, 0}<0$.

We consider the solution $\left(\psi_{1}, \psi_{2}\right)=\left(\psi_{1}^{-}, \psi_{2}^{-}\right)$. The probability constraints imply

$$
\begin{aligned}
& g_{a, 1}<-\sqrt{g_{a, 1}^{2}-4 g_{a, 2} g_{a, 0}}<2 \mu^{-1} g_{a, 2}+g_{a, 1}, \\
& g_{b, 1}>-\sqrt{g_{b, 1}^{2}-4 g_{b, 2} g_{b, 0}}>2 \mu^{-1} g_{b, 2}+g_{b, 1} .
\end{aligned}
$$

From Eq. (42b) we have $2 \mu^{-1} g_{b, 2}+g_{b, 1}<0$, giving

$$
g_{b, 0}+\mu^{-1} g_{b, 1}+\mu^{-2} g_{b, 2}<0,
$$

which cannot hold since its left side equals $\mu \omega \lambda_{a}^{\dagger}$. Thus, in this region, no physical solution of $\left(\psi_{1}, \psi_{2}\right)=\left(\psi_{1}^{-}, \psi_{2}^{-}\right)$exists.

(iv.4) Suppose $\lambda_{a}^{\dagger}<\lambda_{a}$ for $a \in\{1,2\}$, then $c_{a, 2}<0$. For the solution $\left(\psi_{1}, \psi_{2}\right)=\left(\psi_{1}^{+}, \psi_{2}^{+}\right)$, we must have $g_{a, 1}>0$ and $g_{a, 0}<0$ for $a \in\{1,2\}$. Similar to the discussions in the case (iv.3), we have that the sufficient condition for an equilibrium point is $g_{a, 0}<0$ for $a \in\{1,2\}$. The solution $\left(\psi_{1}, \psi_{2}\right)=$ $\left(\psi_{1}^{-}, \psi_{2}^{-}\right)$is nonphysical-see the discussion in (iv.3).

\section{B. Stability analysis}

The starting point to study the stability of the equilibrium points is the Jacobian matrix $J$ of the 2D mean-field system, whose entries are

$$
\begin{aligned}
J_{11}= & -1+\lambda_{1}^{\dagger} \omega \mu\left(1-2 x \psi_{1}\right) \psi_{2}, \\
& +\lambda_{1} \omega\left(1-2 \mu \psi_{1}\right)\left(1-\mu \psi_{2}\right), \\
J_{12}= & \omega \mu\left(\lambda_{1}^{\dagger}-\lambda_{1}\right)\left(1-\mu \psi_{1}\right) \psi_{1}, \\
J_{21}= & \omega \mu\left(\lambda_{2}^{\dagger}-\lambda_{2}\right)\left(1-\mu \psi_{2}\right) \psi_{2}, \\
J_{22}= & -1+\lambda_{2}^{\dagger} \omega \mu\left(1-2 \mu \psi_{2}\right) \psi_{1} \\
& +\lambda_{2} \omega\left(1-2 \mu \psi_{2}\right)\left(1-\mu \psi_{1}\right) .
\end{aligned}
$$

We analyze the stability of the different classes of equilibrium points as discussed in Sec. III A.

(i) Epidemic free. For $\left(\psi_{1}, \psi_{2}\right)=(0,0)$, the Jacobian matrix is

$$
J=\left(\begin{array}{cc}
-1+\lambda_{1} \omega & 0 \\
0 & -1+\lambda_{2} \omega
\end{array}\right)
$$

The equilibrium point is stable for $\lambda_{a}<\omega^{-1}$ for $a \in\{1,2\}$.

(ii) Partial infection of epidemic 1. In this case, we have

$$
\left(\psi_{1}, \psi_{2}\right)=\left(\frac{\lambda_{1} \omega-1}{\mu \lambda_{1} \omega}, 0\right)
$$


and $J_{21}=0$, so the Jacobian is upper triangular, whose eigenvalues are simply the diagonal entries:

$$
\begin{aligned}
& J_{1,1}=1-\lambda_{1} \omega, \\
& J_{2,2}=-1+\frac{\lambda_{2}}{\lambda_{1}}+\lambda_{2}^{\dagger} \frac{\lambda_{1} \omega-1}{\lambda_{1}} .
\end{aligned}
$$

The equilibrium point is stable for $J_{1,1}<0$ and $J_{2,2}<0$, i.e.,

$$
\begin{gathered}
\lambda_{1}>\omega^{-1}, \\
\lambda_{2}-\lambda_{2}^{\dagger}-\lambda_{1}+\omega \lambda_{1} \lambda_{2}^{\dagger}<0 .
\end{gathered}
$$

(iii) Partial infection of epidemic 2. For this type of equilibrium point, we have

$$
\left(\psi_{1}, \psi_{2}\right)=\left(0, \frac{\lambda_{2} \omega-1}{\mu \lambda_{2} \omega}\right) .
$$

It is stable under the following conditions:

$$
\begin{gathered}
\lambda_{2}>\omega^{-1}, \\
\lambda_{1}-\lambda_{1}^{\dagger}-\lambda_{2}+\omega \lambda_{2} \lambda_{1}^{\dagger}<0 .
\end{gathered}
$$

(iv) Coexistence. Suppose we have $\psi_{a} \neq 0$ for $a \in\{1,2\}$. Substituting Eqs. (17) into Eqs. (44), the Jacobian matrix has entries

$$
\begin{aligned}
& J_{11}=\mu \psi_{1} /\left(\mu \psi_{1}-1\right), \\
& J_{12}=\omega \mu\left(\lambda_{1}^{\dagger}-\lambda_{1}\right)\left(1-\mu \psi_{1}\right) \psi_{1}, \\
& J_{21}=\omega \mu\left(\lambda_{2}^{\dagger}-\lambda_{2}\right)\left(1-\mu \psi_{2}\right) \psi_{2}, \\
& J_{22}=\mu \psi_{2} /\left(\mu \psi_{2}-1\right) .
\end{aligned}
$$

A necessary and sufficient condition for a two-dimensional matrix to have two negative eigenvalues is to have a negative trace $(\operatorname{tr}(J)<0)$ but a positive determinant $(\operatorname{det}(J)>0)$. Since $\mu \psi_{a}-1<0$, the negativity of the trace always holds. The stability of a equilibrium point in this class is fully determined by the determinant. It is stable when $\operatorname{det}(J)>0$ and unstable when $\operatorname{det}(J)>0$. The stable condition from the determinant is

$$
\begin{aligned}
\operatorname{det}(J)= & \frac{\mu^{2} \psi_{a} \psi_{b}}{\left(1-\mu \psi_{a}\right)\left(1-\mu \psi_{b}\right)} \\
& -\omega^{2} \mu^{2}\left(\lambda_{a}^{\dagger}-\lambda_{a}\right)\left(\lambda_{b}^{\dagger}-\lambda_{b}\right) \\
& \times\left(1-\mu \psi_{a}\right)\left(1-\mu \psi_{b}\right) \psi_{a} \psi_{b}>0 .
\end{aligned}
$$

Let $z=\left(1-\mu \psi_{a}\right)\left(1-\mu \psi_{b}\right)$, the inequality can be written as

$$
\frac{1}{z}>\omega^{2}\left(\lambda_{a}^{\dagger}-\lambda_{a}\right)\left(\lambda_{b}^{\dagger}-\lambda_{b}\right) z .
$$

Equations (17) can be rearranged as

$$
\begin{aligned}
& \lambda_{1}^{\dagger} \omega\left(1-\mu \psi_{1}\right)=1+\left(\lambda_{1}^{\dagger}-\lambda_{1}\right) \omega z, \\
& \lambda_{2}^{\dagger} \omega\left(1-\mu \psi_{2}\right)=1+\left(\lambda_{2}^{\dagger}-\lambda_{2}\right) \omega z .
\end{aligned}
$$

Multiplying the above two equations, we get

$$
d_{2} z^{-2}+d_{1} z^{-1}+d_{0}=0,
$$

where

$$
\begin{aligned}
& d_{2}=1, \\
& d_{1}=\omega\left(\lambda_{1}^{\dagger}-\lambda_{1}+\lambda_{2}^{\dagger}-\lambda_{2}-\omega \lambda_{1}^{\dagger} \lambda_{2}^{\dagger}\right), \\
& d_{0}=\omega^{2}\left(\lambda_{1}^{\dagger}-\lambda_{1}\right)\left(\lambda_{2}^{\dagger}-\lambda_{2}\right) .
\end{aligned}
$$

Multiplying both sides of Eq. (55) by $z$ and substituting the result into Eq. (53), we obtain

$$
\frac{1}{z}>-\frac{d_{1}}{2}
$$

That is, an equilibrium point is stable if and only if Eq. (57) holds and is unstable otherwise. It remains to find the solutions of Eq. (55) to verify whether Eq. (57) is satisfied.

If the condition $\lambda_{b}^{\dagger}=\lambda_{b}$ holds for one or both values of $b \in\{1,2\}$, then $d_{0}=0$. In this case, we have $z^{-1}=-d_{1} / d_{2}$ and Eq. (53) implies the solution is stable for $d_{1}<0$.

For $\lambda_{a}^{\dagger} \neq \lambda_{b}$ for any $a \in\{1,2\}$, from Eq. (55), we see that $1 / z$ has two solutions

$$
\left(\frac{1}{z}\right)^{ \pm}=\frac{-d_{1} \pm \sqrt{d_{1}^{2}-4 d_{2} d_{0}}}{2 d_{2}} .
$$

Since we have a pair of solutions for $\left(\psi_{1}, \psi_{2}\right)$ as in Eq. (22), the following hold:

$$
\begin{aligned}
& \left(\frac{1}{z}\right)^{+}=\frac{1}{\left(1-\mu \psi_{a}^{+}\right)\left(1-\mu \psi_{b}^{+}\right)}, \\
& \left(\frac{1}{z}\right)^{-}=\frac{1}{\left(1-\mu \psi_{a}^{-}\right)\left(1-\mu \psi_{b}^{-}\right)} .
\end{aligned}
$$

Substituting Eq. (58) into Eq. (57), we have

$$
\pm \sqrt{d_{1}^{2}-4 d_{2} d_{0}} \geqslant 0 .
$$

We see that, given $d_{1}^{2}-4 d_{2} d_{0}>0$, the solution $\left(\psi_{a}^{+}, \psi_{b}^{+}\right)$is always stable, while $\left(\psi_{a}^{-}, \psi_{b}^{-}\right)$is always unstable. It remains to check the validity of the inequality $d_{1}^{2}-4 d_{2} d_{0}>0$. After some algebra, we have

$$
\begin{aligned}
d_{1}^{2}-4 d_{2} d_{0}= & g_{1,1}^{2}-4 g_{1,2} g_{1,0}=g_{2,1}^{2}-4 g_{2,2} g_{2,0} \\
= & \omega \mu^{2}\left(\lambda_{1}^{\dagger}-\lambda_{1}+\lambda_{2}^{\dagger}-\lambda_{2}-\omega \lambda_{1}^{\dagger} \lambda_{2}^{\dagger}\right)^{2} \\
& -4 \omega \mu^{2}\left(\lambda_{1}^{\dagger}-\lambda_{1}\right)\left(\lambda_{2}^{\dagger}-\lambda_{2}\right) .
\end{aligned}
$$

Thus, the inequalities $d_{1}^{2}-4 d_{2} d_{0}>0$ and $g_{a, 1}^{2}-4 g_{a, 2} g_{a, 0}>$ 0 are equivalent to each other for $a \in\{1,2\}$.

\section{Phase diagrams}

With full knowledge about the equilibrium points and their stability, we can obtain the phase diagram of the reduced mean-field system. Define the following set of functions:

$$
\begin{aligned}
s_{a, 0}= & \lambda_{b}+\lambda_{a}^{\dagger}-\lambda_{a}-\omega \lambda_{b} \lambda_{a}^{\dagger}, \\
s_{a, 1}= & \lambda_{b}^{\dagger}-\lambda_{b}-\lambda_{a}^{\dagger}+\lambda_{a}+2 \omega \lambda_{b} \lambda_{a}^{\dagger}-\omega \lambda_{a}^{\dagger} \lambda_{b}^{\dagger}, \\
s_{a, 2}= & \lambda_{b}^{\dagger}-\lambda_{b}, s_{a, 3}=\lambda_{a}-\omega^{-1}, \\
s_{\Delta}= & \left(\lambda_{1}^{\dagger}-\lambda_{1}+\lambda_{2}^{\dagger}-\lambda_{2}-\omega \lambda_{1}^{\dagger} \lambda_{2}^{\dagger}\right)^{2} \\
& -4\left(\lambda_{1}^{\dagger}-\lambda_{1}\right)\left(\lambda_{2}^{\dagger}-\lambda_{2}\right),
\end{aligned}
$$


for $a, b \in\{1,2\}$ and $a \neq b$. The distinct phase regions can be defined via various inequalities among these functions.

(i) Epidemic free. The solution $\left(\psi_{1}, \psi_{2}\right)=(0,0)$ is stable for $s_{a, 3}<0$ for both $a \in\{1,2\}$.

(ii) Partial infection of epidemic 1 . The phase has a stable equilibrium point

$$
\left(\psi_{1}, \psi_{2}\right)=\left(\frac{\lambda_{1} \omega-1}{\mu \lambda_{1} \omega}, 0\right) .
$$

Combining the probability constraints and the stability analysis, we obtain the phase region as

$$
s_{2,0}>0, s_{1,3}>0 .
$$

(iii) Partial infection of epidemic 2. The phase is characterized by

$$
\left(\psi_{1}, \psi_{2}\right)=\left(0, \frac{\lambda_{2} \omega-1}{\mu \lambda_{2} \omega}\right) .
$$

The phase region is given by

$$
s_{1,0}>0, s_{2,3}>0 .
$$

(iv) Coexistence. In this region, there is an equilibrium point with both $\psi_{1}$ and $\psi_{2}$ nonzero, corresponding to the case of double epidemic outbreaks. For cooperative coevolution, i.e., $\lambda_{a}^{\dagger}>\lambda_{a}$ for $a \in\{1,2\}$, a point in the parameter space belongs to this phase if

$$
s_{a, 0}>0, s_{a, 1}<0, s_{a, 2}>0, s_{\Delta}>0,
$$

or

$$
s_{a, 0}<0, s_{a, 2}>0,
$$

for both $a \in\{1,2\}$. When coevolution is not cooperative, the coexistence region is given by

$$
s_{a, 0}<0
$$

for both $a \in\{1,2\}$. We have verified that the case of $\lambda_{a}=\lambda_{a}^{\dagger}$ for one or both $a \in\{1,2\}$ is well covered by this inequality.

( $\mathrm{i} \cap \mathrm{iv}$ ). Hysteresis region 1. A hysteresis region appears when there are two stable equilibrium points and one unstable equilibrium point in between. The stability analysis indicates that the solution $\left(\psi_{1}^{+}, \psi_{2}^{+}\right)$is always stable while $\left(\psi_{1}^{-}, \psi_{2}^{-}\right)$is unstable. In addition to these two equilibrium points, a third stable solution is necessary for a hysteresis to arise. This is only possible when region (iv) overlaps with regions (i), (ii), and (iii). Checking the equilibrium points and their stability, we find that a hysteresis region exists only when the inequality $\lambda_{a}^{\dagger}>\lambda_{a}$ holds for $a \in\{1,2\}$. The region where (i) and (iv) overlap is

$$
s_{a, 0}>0, s_{a, 1}<0, s_{a, 2}>0, s_{a, 3}<0, s_{\Delta}>0,
$$

where the first inequality $s_{a, 0}>0$ can in fact be implied by the other inequalities. Since $g_{1,1}+g_{2,1}<0$, we have

$$
\lambda_{1}^{\dagger}+\lambda_{2}^{\dagger}-\omega \lambda_{1}^{\dagger} \lambda_{2}^{\dagger}<0,
$$

which further implies $\omega \lambda_{1}^{\dagger}>1$ and $\omega \lambda_{2}^{\dagger}>1$. Since $s_{a, 3}<0$, we have

$$
s_{a, 0}=\lambda_{b}\left(1-\omega \lambda_{a}^{\dagger}\right)+\lambda_{a}^{\dagger}-\lambda_{a}>\omega^{-1}-\lambda_{a}>0 .
$$

Altogether, the region is given by

$$
s_{a, 1}<0, s_{a, 2}>0, s_{a, 3}<0, s_{\Delta}>0
$$

for $a \in\{1,2\}$.

(ii $\cap$ iv). Hysteresis region 2 . This region is where (ii) and (iv) overlap, which is bounded by the inequalities

$$
s_{a, 0}>0, s_{a, 1}<0, s_{a, 2}>0, s_{1,3}>0, s_{2,3}<0, s_{\Delta}>0
$$

for $a \in\{1,2\}$.

(iii $\cap$ iv). Hysteresis region 3. Similarly, the region where (iii) and (iv) overlap is bounded by

$$
s_{a, 0}>0, s_{a, 1}<0, s_{a, 2}>0, s_{1,3}<0, s_{2,3}>0, s_{\Delta}>0
$$

for $a \in\{1,2\}$.

\section{Types of phase transition}

A phase transition occurs when a point in the parameter space crosses a boundary between two neighboring phase regions. Depending on different combinations of phase-region pairs, the resulting phase transitions can be characteristically distinct. To be concrete, we focus on the phase transitions in the $\lambda_{1}-\lambda_{2}$ plane with fixed values of $\lambda_{1}^{\dagger}$ and $\lambda_{2}^{\dagger}$. Both continuous and discontinuous phase transitions can arise, as we will show below.

(i) $\rightleftharpoons$ (ii): We have that the equations $s_{1,0}=0$ and $s_{2,0}=0$ intersect at the point $\left(\lambda_{1}, \lambda_{2}\right)=\left(\omega^{-1}, \omega^{-1}\right)$, so the two phases are separated by the line $s_{1,3}=0$ in the $\lambda_{1}-\lambda_{2}$ plane. When approaching the line $s_{1,3}=0$ from phase (ii), the equilibrium point

$$
\left(\psi_{1}, \psi_{1}\right)=\left(\frac{\lambda_{1} \omega-1}{\mu \lambda_{1} \omega}, 0\right)
$$

approaches $\left(\psi_{1}, \psi_{1}\right)=(0,0)$. As a result, a continuous phase transition arises.

(i) $\rightleftharpoons$ (iii): Similar to the preceding case, the phase transition is continuous.

(ii) $\rightleftharpoons$ (iv) $\backslash$ (ii $\cap$ iv): The two phases are separated by the line $s_{2,0}=0$. When the stable equilibrium point $\left(\psi_{1}^{+}, \psi_{2}^{+}\right)$in Eq. (21) approaches the line, for $s_{2,1}>0$ we have

$$
\left(\psi_{1}^{+}, \psi_{2}^{+}\right) \rightarrow\left(\frac{\lambda_{1} \omega-1}{\mu \lambda_{1} \omega}, 0\right)
$$

generating a continuous phase transition. Otherwise $\left(s_{2,1}<\right.$ $0)$, we have

$$
\left(\psi_{1}^{+}, \psi_{2}^{+}\right) \rightarrow\left(-\frac{g_{1,2}+g_{2,1}}{g_{1,2}},-\frac{g_{2,1}}{g_{2,2}}\right),
$$

so the phase transition is discontinuous. It remains to discuss the sign of $s_{2,1}$. Substituting $s_{2,0}=0$ into $s_{2,1}$, we get

$$
s_{2,1}=\lambda_{1}^{\dagger}+\omega \lambda_{1} \lambda_{2}^{\dagger}-\omega \lambda_{1}^{\dagger} \lambda_{2}^{\dagger} .
$$

First consider the case where the coevolution dynamics are not cooperative, i.e., $\lambda_{a} \geqslant \lambda_{a}^{\dagger}$ for at least one of $a \in\{1,2\}$. In this case, the region (ii $\cap$ iv) is empty. Suppose $\lambda_{1} \geqslant \lambda_{1}^{\dagger}$, it can be immediately seen that $s_{2,1}>0$. For $\lambda_{2} \geqslant \lambda_{2}^{\dagger}$, we have $s_{2,0}>0$, implying $\omega_{2} \lambda_{2}^{\dagger} \leqslant 1$ and consequently $s_{2,1}>0$. 
Now consider the case of cooperative coevolution dynamics, where a point in the region (ii $\cap$ iv) satisfies $s_{2,1}<0$. Further, we can prove that, if a point is in the region (iv) $\backslash$ (ii $\cap$ iv), then $s_{2,1}>0$. This is accomplished by showing that if a point has $s_{2,1}<0$ then it must be in the region (ii $\cap$ iv). Notice that the equations $s_{2,1}=0, s_{s_{2,0}}=0$, and $s_{\Delta}=0$ intersect at the point

$$
\left(\lambda_{1}, \lambda_{2}\right)=\left(\lambda_{1}^{\dagger}-\frac{\lambda_{1}^{\dagger}}{\omega \lambda_{2}^{\dagger}}, 2 \lambda_{1}^{\dagger}+\lambda_{2}^{\dagger}-\omega \lambda_{1}^{\dagger} \lambda_{2}^{\dagger}-\frac{\lambda_{1}^{\dagger}}{\omega \lambda_{2}^{\dagger}}\right)
$$

in the $\lambda_{1}-\lambda_{2}$ plane. Since $s_{2,1}$ is an increasing function of $\lambda_{1}$ along $s_{2,0}=0$, as can be seen from Eq. (79), we have that, if a point in the line defined by $s_{2,0}=0$ in the $\lambda_{1}-\lambda_{2}$ plane has $\lambda_{1}<\lambda_{1}^{\dagger}-\lambda_{1}^{\dagger} / \omega \lambda_{2}^{\dagger}$, it will satisfy $s_{2,1}<0$. Furthermore, since $\lambda_{1}>\omega^{-1}$, the inequality $\lambda_{1}<\lambda_{1}^{\dagger}-\lambda_{1}^{\dagger} / \omega \lambda_{2}^{\dagger}$ implies

$$
\lambda_{1}^{\dagger}+\lambda_{2}^{\dagger}<\omega \lambda_{1}^{\dagger} \lambda_{2}^{\dagger} \text {. }
$$

Along the line $s_{2,0}=0, s_{1,0}$ can be written as

$$
s_{1,0}=\left(\omega \lambda_{1}^{\dagger} \lambda_{2}^{\dagger}-\lambda_{1}^{\dagger}-\lambda_{2}^{\dagger}\right) \lambda_{1}+\lambda_{1}^{\dagger}+\lambda_{2}^{\dagger}-\omega \lambda_{1}^{\dagger} \lambda_{2}^{\dagger},
$$

which is an increasing function of $\lambda$. Since the curves $s_{1,0}=0$ and $s_{2,0}=0$ intersect at the point $\left(\lambda_{1}, \lambda_{2}\right)=\left(\omega^{-1}, \omega^{-1}\right)$, we have $s_{1,0}=0$. We thus have $s_{1,0}>0$ for $\lambda_{1}>\omega^{-1}$. Similarly, along the line $s_{2,0}=0$, we have

$$
\begin{aligned}
s_{1,1} & =-\lambda_{1}^{\dagger}+\omega \lambda_{1} \lambda_{2}^{\dagger}+2 \omega \lambda_{2} \lambda_{1}^{\dagger}-\omega \lambda_{1}^{\dagger} \lambda_{2}^{\dagger} \\
& <-2 \lambda_{1}^{\dagger}+2 \omega \lambda_{2} \lambda_{1}^{\dagger}<0 .
\end{aligned}
$$

The first inequality is the result of $\lambda_{1}<\lambda_{1}^{\dagger}-\lambda_{1}^{\dagger} / \omega \lambda_{2}^{\dagger}$ and the second inequality is due to the fact $\lambda_{2}<\omega^{-1}$ along the line $s_{2,0}=0$ for $\lambda_{1}>\omega^{-1}$. Last, a point in region (ii) can always make $s_{\Delta}>0$ if it is sufficiently close to the line $s_{2,0}=0$.

To summarize, if a point in region (ii) has $s_{2,1}<0$ near the phase boundary $s_{2,0}=0$, then all the conditions under which the point is in region (ii $\cap$ iv) hold. Thus, if the point is in the region (iv) $\backslash$ (ii $\cap$ iv), then we have $s_{2,1}>0$, which makes the phase transition continuous.

(iii) $\rightleftharpoons$ (iv) $\backslash$ (iii $\cap$ iv). Following a similar treatment to the preceding case, we have that the phase transition is continuous.

(ii $\cap$ iv) $\rightarrow$ (iv). The two phase are separated by the line $s_{2,0}=0$. As discussed in the case of the (ii) $\rightleftharpoons(\mathrm{iv}) \backslash$ (ii $\cap$ iv) transition, since $s_{2,1}<0$ holds near the phase boundary, the behavior of the coexistence solution is determined by Eq. (78) when approaching the phase boundary, resulting in a discontinuous phase transition.

With discussions similar to those in the (ii $\cap$ iv) $\rightarrow$ (iv) case, we find that all transitions as a result of entering or leaving the hysteresis region are of the discontinuous type, due to the fact that, in the hysteresis region, the inequality $s_{a, 1}<0$ holds. The discontinuous transitions include (ii $\cap$ iv) $\rightarrow$ (iv), (iii $\cap$ iv) $\rightarrow$ (iv), (i $\cap$ iv) $\rightarrow$ (iv), (ii $\cap$ iv) $\rightarrow$ (ii) $\backslash$ (ii $\cap$ iv), (iii $\cap$ iv) $\rightarrow$ (iii) $\backslash($ iii $\cap$ iv) and (i $\cap$ iv) $\rightarrow$ (i) $\backslash(\mathrm{i} \cap \mathrm{iv})$.

The tricritical points that separate the continuous from the discontinuous transition lie in the boundaries of the hysteresis region where $q_{a, 1}=0$ holds for either $a \in\{1,2\}$. One such point is given by Eq. (80). The second tricritical point can be obtained similarly as

$$
\left(\lambda_{1}, \lambda_{2}\right)=\left(2 \lambda_{2}^{\dagger}+\lambda_{1}^{\dagger}-\omega \lambda_{1}^{\dagger} \lambda_{2}^{\dagger}-\frac{\lambda_{2}^{\dagger}}{\omega \lambda_{1}^{\dagger}}, \lambda_{2}^{\dagger}-\frac{\lambda_{2}^{\dagger}}{\omega \lambda_{1}^{\dagger}}\right) .
$$

\section{E. Conditions on $\lambda_{a}^{\dagger}$ for hysteresis}

For fixed values of $\lambda_{1}^{\dagger}$ and $\lambda_{2}^{\dagger}$, a hysteresis can arise in the $\lambda_{1}-\lambda_{2}$ plane. To determine these values, we first note that a hysteresis is possible only when the coevolution dynamics are cooperative, i.e., $\lambda_{a}^{\dagger}>\lambda_{a}$ for both $a \in\{1,2\}$. A point in the hysteresis region must satisfy the inequality $g_{1,1}+g_{2,1}<0$. Consequently, we have

$$
\lambda_{1}^{\dagger}+\lambda_{2}^{\dagger}-\omega \lambda_{1}^{\dagger} \lambda_{2}^{\dagger}<0
$$

which provides a necessary condition for a hysteresis to arise. We can show that this is also sufficient to guarantee the occurrence of a hysteresis. In particular, suppose inequality Eq. (102) holds. Since $s_{1,0}=0$ and $s_{2,0}=0$ intersect at $\left(\lambda_{1}, \lambda_{2}\right)=\left(\omega^{-1}, \omega^{-1}\right)$ in the $\lambda_{a}-\lambda_{b}$ plane, there is a neighborhood near $\left(\omega^{-1}, \omega^{-1}\right)$ in which the inequalities $s_{1,0}>0$ and $s_{2,0}>0$ hold. At the point $\left(\lambda_{a}, \lambda_{b}\right)=\left(\omega^{-1}, \omega^{-1}\right)$, we have

$$
s_{1,1}=s_{2,1}=\lambda_{1}^{\dagger}+\lambda_{2}^{\dagger}-\omega \lambda_{1}^{\dagger} \lambda_{2}^{\dagger}<0 .
$$

It remains to check whether the inequality $s_{\Delta}>0$ holds. Let $\omega \lambda_{a}^{\dagger} \lambda_{b}^{\dagger}=\lambda_{a}^{\dagger}+\lambda_{b}^{\dagger}+\epsilon$, where $\epsilon>0$ is a constant. Then at the point $\left(\lambda_{a}, \lambda_{b}\right)=\left(\omega^{-1}, \omega^{-1}\right)$, we have

$$
s_{\Delta}=\left(2 \omega^{-1}+\epsilon\right)^{2}-4\left(\epsilon \omega^{-1}+\omega^{-2}\right)=\epsilon>0 .
$$

We thus have that a hysteresis region exists in the $\lambda_{1}-\lambda_{2}$ plane if and only if Eq. (85) holds.

\section{F. Summary of the phase diagrams}

Based on the results of the above analysis, we obtain the structure of the analytically predicted phase diagram, which can be described, as follows.

(i) Epidemic free region. In this region, Eq. (11) has the equilibrium point $\left(\psi_{1}, \psi_{2}\right)=(0,0)$, indicating extinction of both epidemics. The solution is stable for $s_{a, 3}<0(a \in$ $\{1,2\})$. The phase boundary $\lambda_{a}=\omega^{-1}$ is also the outbreak threshold for the classic SIS model with a single epidemic.

(ii) Partial infection of epidemic 1. In this phase, an outbreak occurs for the epidemic 1 but not for 2 . The equilibrium point is given by

$$
\left(\psi_{1}, \psi_{2}\right)=\left(\frac{\lambda_{1} \omega-1}{\mu \lambda_{1} \omega}, 0\right),
$$

which is stable for $s_{2,0}>0$ and $s_{1,3}>0$, where the latter gives $\lambda_{1}>\omega^{-1}$, indicating that epidemic 1 can have an outbreak independently. Similarly, $s_{2,0}>0$ implies $\lambda_{2}<\lambda_{1}$ and

$$
\lambda_{2}^{\dagger}<\left(\lambda_{1}-\lambda_{2}\right) /\left(\omega \lambda_{1}-1\right)
$$

stipulating that $\lambda_{2}^{\dagger}$ cannot be too large, such that the outbreak of epidemic 1 result in an outbreak in epidemic 2 .

(iii) Partial infection of epidemic 2. Analogous to (ii), this phase is defined by

$$
\left(\psi_{1}, \psi_{2}\right)=\left(0, \frac{\lambda_{2} \omega-1}{\mu \lambda_{2} \omega}\right),
$$

which is stable for $s_{1,0}>0$ and $s_{2,3}>0$. 
(iv) Coexistence. In this region the reduced system has a stable equilibrium point with both $\psi_{1}$ and $\psi_{2}$ nonzero, leading to a simultaneous outbreak of two epidemics. The stable equilibrium points are

$$
\left(\psi_{1}, \psi_{2}\right)=\left(\frac{-s_{1,1} \pm \sqrt{s_{\Delta}}}{2 \mu \omega \lambda_{1}^{\dagger} s_{1,2}}, \frac{-s_{2,1} \pm \sqrt{s_{\Delta}}}{2 \mu \omega \lambda_{2}^{\dagger} s_{2,2}}\right) .
$$

For cooperative coevolution, i.e., $\lambda_{a}^{\dagger}>\lambda_{a}$ for $a \in\{1,2\}$, we have $s_{a, 2}>0$. A point in the parameter space belongs to this phase if it further satisfies either

$$
s_{a, 0}>0, s_{a, 1}<0, s_{\Delta}>0,
$$

or

$$
s_{a, 0}<0
$$

for $a \in\{1,2\}$. The former case is where region (iv)overlaps with regions (i), (ii), and (iii). As a result, hystereses can arise. For competitive or asymmetric coevolution, the coexistence region is given by $s_{a, 0}<0$ for $a \in\{1,2\}$.

Now we discuss the conditions for observing the coexistence phase in more detail to get an intuitive picture. For the cooperative case, the boundaries of the coexistence region are relatively complex, and we consider the degenerate case of $\lambda_{1}=\lambda_{2}$ and $\lambda_{1}^{\dagger}=\lambda_{2}^{\dagger}$. For $s_{a, 0}<0$, we have $\omega \lambda_{1}=\omega \lambda_{2}>1$ so that both epidemics are able to have an outbreak by themselves. On the contrary, for $s_{a, 0}>0$ so that neither epidemic can have an outbreak by itself, we have $s_{a, 1}<0$ and $\lambda_{1}^{\dagger}>2 \lambda_{1}$. The condition $s_{\Delta}>0$ requires

$$
\left(\omega \lambda_{1}^{\dagger}\right)^{2}>4\left(\omega \lambda_{1}^{\dagger}-\omega \lambda_{1}\right)
$$

and $\lambda_{1}^{\dagger}>2 \lambda_{1}$, leading to the necessary condition $\omega \lambda_{1}^{\dagger}>2$. In this case, the interaction transmission rate must at least double the threshold value of classic SIS outbreak to have coexistence.

For the competitive case, since $s_{a, 0}<0$, we have

$$
s_{1,0}+s_{2,0}=\lambda_{1}^{\dagger}\left(1-\omega \lambda_{2}\right)+\lambda_{2}^{\dagger}\left(1-\omega \lambda_{2}\right)<0 .
$$

The above inequality implies that at least one of $\lambda_{a}$, say $\lambda_{1}$, must satisfy $\omega \lambda_{1}>1$. Actually, we must also have $\omega \lambda_{2}>1$ to observe the coexistence phase. Suppose $\omega \lambda_{2}<1$, from $\omega s_{1,0}<0$, we have

$$
\begin{aligned}
& \omega \lambda_{1}-\omega \lambda_{2}+\omega \lambda_{2}^{\dagger}\left(1-\omega \lambda_{1}\right)<0 \\
\Rightarrow & \omega \lambda_{2}^{\dagger}>\frac{\omega \lambda_{2}-\omega \lambda_{1}}{1-\omega \lambda_{1}}>1>\omega \lambda_{2},
\end{aligned}
$$

which contradicts with the assumption that the model is competitive. Consequently, to observe the coexistence of two epidemics for the competitive case, a necessary condition is that each of the two epidemics can have an outbreak by itself. In addition, $s_{a, 0}<0$ implies

$$
\lambda_{a}^{\dagger}>\frac{\lambda_{a}-\lambda_{b}}{1-\omega \lambda_{b}} .
$$

That is to say, to have the coexistence phase, the suppression effect from the other epidemic cannot be too strong. For networks with a larger value of $\omega$, the conditions Eq. (97) and $\omega \lambda_{a}>1$ both can be readily satisfied. As a result, the coexistence phase is more likely to be observed in networks with a larger leading eigenvalue $\omega$.

It is also interesting to note that, when the two epidemics have fully mutual exclusion (i.e., $\lambda_{a}^{\dagger}=0$ ), the condition $s_{a, 0}<$ 0 can never be satisfied simultaneously for both $a \in\{1,2\}$. In other words, the coexistence phase can never be observed with fully mutual exclusion, and this phenomenon agrees with the prediction in Ref. [38].

(i $\cap$ iv) Hysteresis region 1. A hysteresis arises when there are two stable equilibrium points and one unstable equilibrium point in between, which occurs when region (iv) overlaps with regions (i), (ii) and (iii). Our analysis reveals that a hysteresis region emerges only for cooperative coevolution. The region where (i) and (iv) overlap is bounded by the inequalities

$$
s_{a, 1}<0, s_{a, 2}>0, s_{a, 3}<0, s_{\Delta}>0
$$

for $a \in\{1,2\}$.

(ii $\cap$ iv) Hysteresis region 2. This is where (ii) and (iv) overlap. Besides the cooperative condition $s_{a, 2}>0$, the region is nonempty if the inequalities

$$
s_{a, 0}>0, s_{a, 1}<0, s_{1,3}>0, s_{2,3}<0, s_{\Delta}>0
$$

hold for $a \in\{1,2\}$.

(iii $\cap$ iv) Hysteresis region 3. Similarly, for cooperative coevolution with $s_{a, 2}>0$, the region where (iii) and (iv) overlap is bounded by

$$
s_{a, 0}>0, s_{a, 1}<0, s_{1,3}<0, s_{2,3}>0, s_{\Delta}>0
$$

for $a \in\{1,2\}$.

The types of phase transitions that occur when crossing a phase boundary are determined by further checking if the stable solution varies continuously (see Sec. III D). We find all possible phase transitions as a result of crossing a hysteresis region are discontinuous, whereas other transitions are continuous. A result revealed by our analysis of the phase diagrams is that the precursor of a discontinuous transition with an abrupt outbreak of at least one epidemic is a hysteresis. Continuous and discontinuous phase transitions are separated by two tricritical points in the $\lambda_{1}-\lambda_{2}$ plane:

$$
\begin{aligned}
& \left(\lambda_{1}, \lambda_{2}\right)=\left(\lambda_{1}^{\dagger}-\frac{\lambda_{1}^{\dagger}}{\omega \lambda_{2}^{\dagger}}, 2 \lambda_{1}^{\dagger}+\lambda_{2}^{\dagger}-\omega \lambda_{1}^{\dagger} \lambda_{2}^{\dagger}-\frac{\lambda_{1}^{\dagger}}{\omega \lambda_{2}^{\dagger}}\right), \\
& \left(\lambda_{1}, \lambda_{2}\right)=\left(2 \lambda_{2}^{\dagger}+\lambda_{1}^{\dagger}-\omega \lambda_{1}^{\dagger} \lambda_{2}^{\dagger}-\frac{\lambda_{2}^{\dagger}}{\omega \lambda_{1}^{\dagger}}, \lambda_{2}^{\dagger}-\frac{\lambda_{2}^{\dagger}}{\omega \lambda_{1}^{\dagger}}\right) .
\end{aligned}
$$

The phase diagram also makes it possible to obtain the conditions in the interaction strengths $\lambda_{1}^{\dagger}$ and $\lambda_{2}^{\dagger}$ for a hysteresis to occur. In Sec. III E, we obtain the necessary and sufficient condition

$$
\lambda_{1}^{\dagger}+\lambda_{2}^{\dagger}-\omega \lambda_{1}^{\dagger} \lambda_{2}^{\dagger}<0
$$

where there is a hysteresis region with $\lambda_{1}<\lambda_{1}^{\dagger}$ and $\lambda_{2}<\lambda_{2}^{\dagger}$.

\section{NUMERICAL VERIFICATION}

We provide a numerical illustration of the analytic prediction on the interplay between discontinuous transitions and hystereses with an Erdős-Rényi graph of size $N=100$ and average degree $\langle k\rangle=4$. The inequality Eq. (102) divides the 

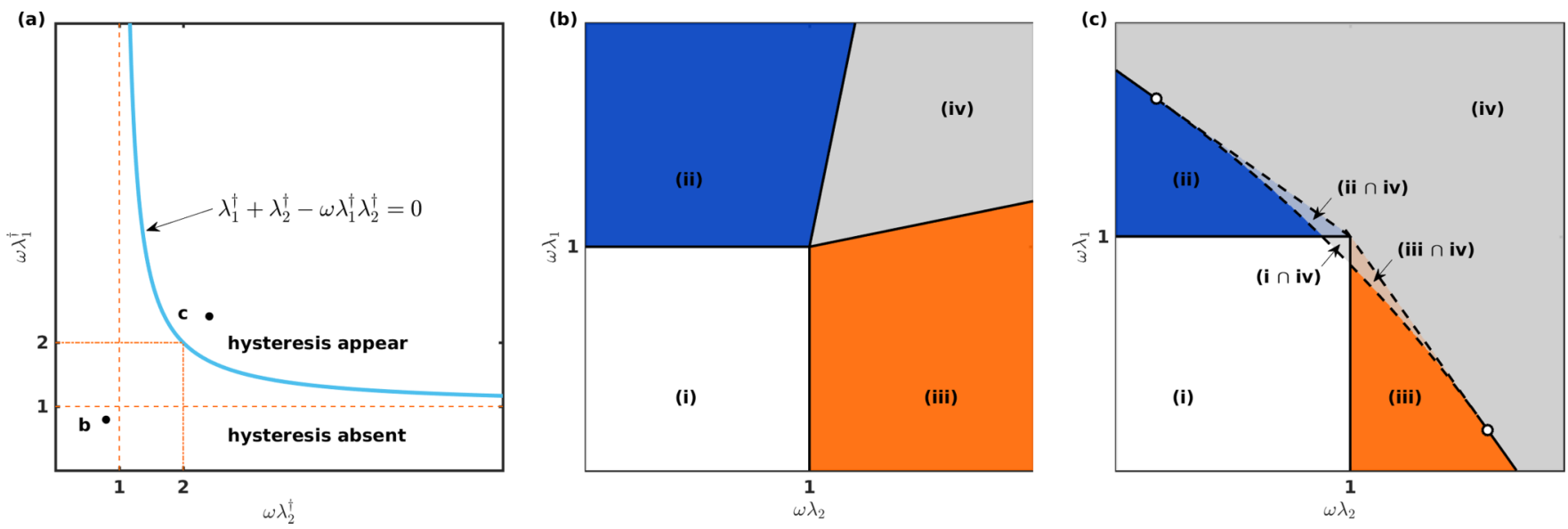

FIG. 1. Phase diagrams of interacting SIS dynamics. For Erdős-Rényi type of random graph of size $N=100$ and average degree $\langle k\rangle=$ 4 , (a) the condition for interactive transmission rates $\lambda_{1}^{\dagger}$ and $\lambda_{2}^{\dagger}$ for hysteresis in the $\lambda_{1}-\lambda_{2}$ plane. The red dashed and dashed-dotted lines correspond, respectively, to $\lambda_{a}=\omega^{-1}$ and $\lambda_{a}=2 \omega^{-1}$ for $a \in\{1,2\}$. (b, c) Phase diagrams with $\lambda_{1}^{\dagger}$ and $\lambda_{2}^{\dagger}$ corresponding to points $b$ and $c$ in (a), respectively. The solid and dashed lines between different phase regions indicate continuous and discontinuous transitions, respectively. The two white circles in panel (c) are the tricritical points separating discontinuous from continuous transitions.

$\lambda_{1}^{\dagger}-\lambda_{2}^{\dagger}$ plane into two regions, as shown in Fig. 1(a). Above the curve defined by

$$
\lambda_{1}^{\dagger}+\lambda_{2}^{\dagger}-\omega \lambda_{1}^{\dagger} \lambda_{2}^{\dagger}=0
$$

a hysteresis region appears while it is absent below. In the limit $\lambda_{a}^{\dagger} \rightarrow \infty$, the curve approaches $\lambda_{b}^{\dagger}=\omega^{-1}$, as shown by the orange dashed lines in Fig. 1(a). Note that the curve avoids the dashed lines for finite $\lambda_{a}^{\dagger}$. Since $\omega^{-1}$ is also the outbreak threshold of the classic SIS model for a single epidemic, a necessary condition for a hysteresis is that $\lambda_{a}^{\dagger}$ must be larger than the classic threshold. A special case is $\lambda_{1}^{\dagger}=\lambda_{2}^{\dagger}$, where Eq. (102) implies that, for a hysteresis to arise, the inequality $\lambda_{1}^{\dagger}=\lambda_{2}^{\dagger}>2 \omega^{-1}$ must hold. That is, the interactive transmission rate must at least twice the classic SIS threshold for a hysteresis to arise, suggesting that networks with a larger leading eigenvalue are more prone to hystereses. Two representative phase diagrams in the $\lambda_{1}-\lambda_{2}$ plane with fixed values of $\lambda_{1}^{\dagger}$ and $\lambda_{2}^{\dagger}$ are shown in Figs. 1(b) and 1(c), corresponding to the points $b$ and $c$ in Fig. 1(a), respectively. For point $b$, no hysteresis can arise for any values of $\left(\lambda_{1}\right.$, $\left.\lambda_{2}\right)$ and the phase transitions between different neighboring phase regions are continuous, as indicated by the solid lines in Fig. 1(b). For point $c$ that is slightly above the hysteresis boundary, region (iv) overlaps with regions (i), (ii), and (iii), where a hysteresis can arise. Crossing into region (iv) from any one of the phase regions ( $\mathrm{i} \cap$ iv), (ii $\cap$ iv), and (iii $\cap$ iv), a discontinuous outbreak transition occurs with some $\psi_{a}$ changing abruptly from zero to a nonzero value. Along the path (i $\cap$ iv) $\rightarrow$ (i), (ii $\cap$ iv) $\rightarrow$ (ii), and (iii $\cap$ iv) $\rightarrow$ (iii), the system displays a discontinuous transition to extinction at which at least one epidemic changes abruptly from a nonzero value to zero. All the phase boundaries with discontinuous transitions are indicated by the dashed lines in Fig. 1(c), where the two tricritical points separating continuous from discontinuous transitions are marked (white circles).

Are the phase diagrams obtained from the reduced mean field equations accurate in comparison with those from the original mean field equations? In the presence of the fluctu- ation terms $R_{a}$, Eq. (11) are exactly equivalent to Eqs. (1). Consider a system of dimension $2 N+2$, which consists of Eqs. (1) and (11). A stable equilibrium point $\left(p_{1, i}, p_{2, i}\right)_{1 \leqslant i \leqslant N}$ of the subsystem Eqs. (1) is also one for the $2 N+2$ system with $\psi_{a}=\alpha^{T} p_{a}$. Consider a stable equilibrium point with which neither epidemic has an outbreak. Substituting $p_{a, 1}=$ $\cdots=p_{a, N}=0$ and $\psi_{a}=0$ into the remainder term (with full expression in Sec. II B), we have $R_{a}=0$ for $a \in\{1,2\}$. In this case the remainder terms can be ignored. Since a zero stable equilibrium point of Eqs. (1) implies the existence of exactly such a point of Eq. (11) (with no remainder terms) and vice versa, any outbreak transition threshold from phase (i) is expected to be exact for Eqs. (1).

There are two cases where the remainder terms do not vanish and can lead to inaccuracies of the analytic prediction. The first case is when Eqs. (1) exhibit a stable equilibrium point at which there is an outbreak for epidemic 1 but extinction for epidemic $2: R_{1}=0$ and $R_{2} \neq 0$. The second case is when Eqs. (1) have a stable equilibrium point with an outbreak for both epidemics: $R_{a} \neq 0$ for $a \in\{1,2\}$. Since the remainder terms are small by construction, they lead to corrections that can be neglected, which have been verified numerically. Especially, for the Erdős-Rényi network in Fig. 1, we solve Eqs. (1) numerically and compare the solutions with the analytic phase diagram obtained from Eq. (11). The values of $\psi_{a}$ obtained from Eqs. (1) in the $\lambda_{1}-\lambda_{2}$ plane are shown in Figs. 2(a)2(d), for $\lambda_{1}^{\dagger}=3.5 \omega^{-1}$ and $\lambda_{2}^{\dagger}=2.5 \omega^{-1}$ [so that (102) is satisfied, guaranteeing a hysteresis]. Since in the hysteresis region there are two stable equilibrium points for each $\psi_{a}$, we plot separately the two solutions for $\psi_{1}$ in Figs. 2(a) and 2(b), and those for $\psi_{2}$ in Figs. 2(c) and 2(d), respectively. The phase diagram from original Eq. (11) is also shown in Fig. 2 by the orange solid and dashed lines for continuous and discontinuous transitions, respectively. Our analytical phase diagram predicts accurately all outbreak transitions: (i) $\rightarrow$ (ii), (i) $\rightarrow$ (iii), (i $\cap$ iv) $\rightarrow$ (iv), (ii $\cap$ iv) $\rightarrow$ (iv), and (iii $\cap$ iv) $\rightarrow$ (iv). However, quantitatively, the predicted extinction transitions (i $\cap$ iv) $\rightarrow$ (i), (ii $\cap$ iv) $\rightarrow$ (ii), and (iii $\cap$ iv) $\rightarrow$ (iii) are less accurate, due to the nonzero remainders $R_{1}$ and $R_{2}$ 

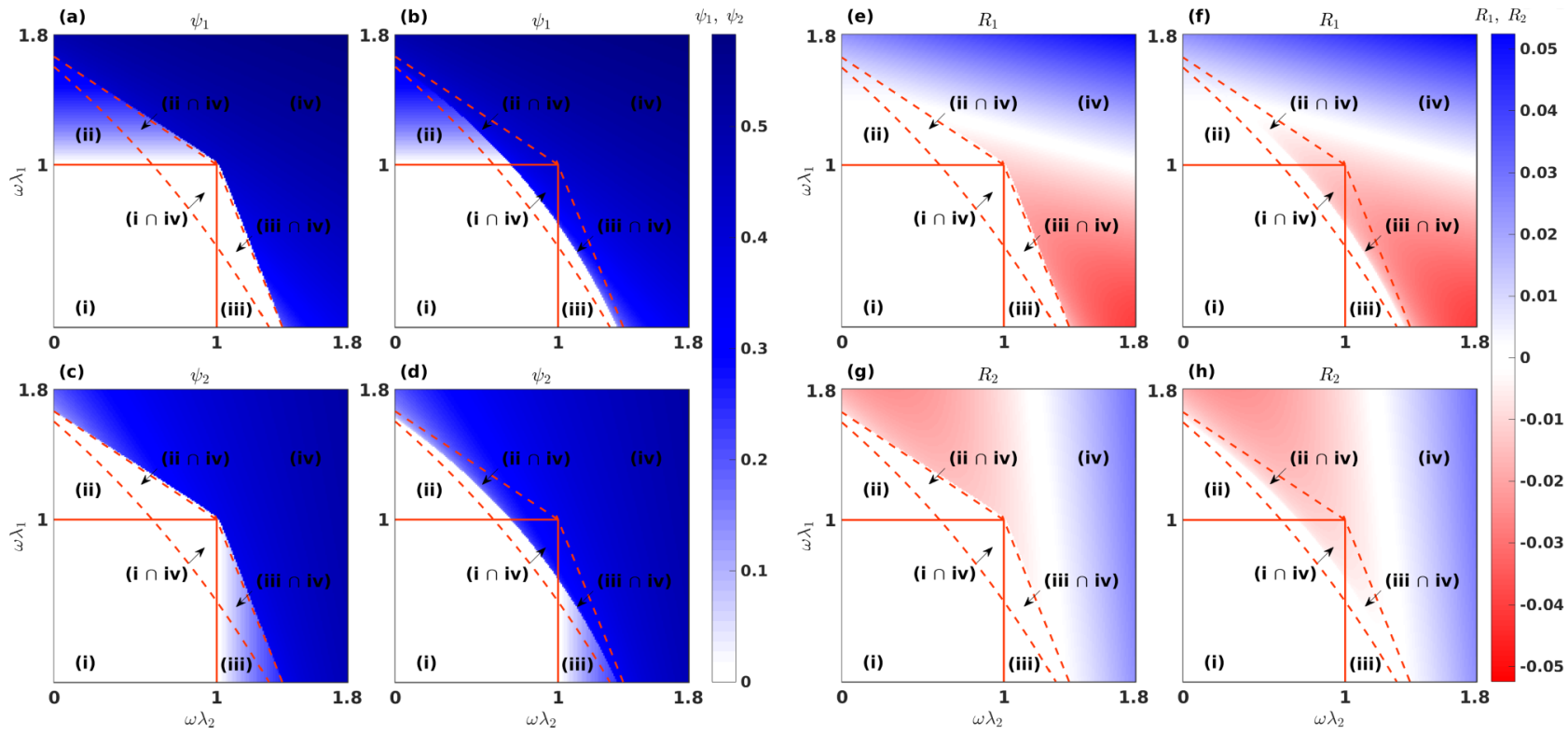

FIG. 2. Validation of analytic predictions. Shown is comparison of the analytically predicted phase diagram with that obtained from the original mean field equations: equilibrium points for color-coded $(\mathrm{a}, \mathrm{b}) \psi_{1}$ and $(\mathrm{c}, \mathrm{d}) \psi_{2}$ values; color-coded values of the remainder terms $R_{1}(\mathrm{e}, \mathrm{f})$ and $R_{2}(\mathrm{~g}, \mathrm{~h})$. The orange lines are the analytically predicted phase boundaries, with solid and dashed lines denoting continuous and discontinuous transitions, respectively.

as a result of the loss of stability of an equilibrium point with an outbreak for both epidemics. The values of the remainders $R_{1}$ and $R_{2}$ at equilibrium are shown in Figs. 2(e)-2(h). The value of $R_{1}$ for the two solutions of $\psi_{1}$ are shown in Figs. 2(e) and 2(f), respectively. Similarly, $R_{2}$ for the two solutions of $\psi_{2}$ are shown in Figs. 2(g) and 2(h), respectively. The predictions are qualitatively correct.

Next we consider tests and validation of our analytic prediction from Eq. (11) for a variety of networks, including synthetic networks with strong and weak degree heterogeneity, and real-world networks. For synthetic networks, we have already shown the results for an ER network. Here we also consider networks generated from the uncorrelated configuration model (UCM) with a power-law degree distribution $p(k) \sim k^{-\beta}$. Specifically, we consider three networks with different degree exponents: (1) PL-2.3 with $\beta=2.3$, (2) PL-3 with $\beta=3$, and (3) PL-4 with $\beta=4$. For empirical networks, we have (4) Dolphins [39], a social network of bottle-nose dolphins; (5) HIV [40], a network of sexual contacts between people involved in the early spread of the human immunodeficiency virus (HIV); (6) Highschool [41], a friendship network between boys in a small high school, and (7) Jazz [42], a collaboration network between Jazz musicians. The networks are downloaded from Ref. [43].

Basic features and parameters of the networks considered are listed in Table I. Note that Highschool is a directed and weighted network. Here we simply take it as undirected by assuming that there is an undirected edge between node $i$ and $j$ if there is at least a directed edge between the two nodes in either direction, with the edge weights ignored.

For all the networks, we set $\lambda_{1}^{\dagger}=3.5 \omega^{-1}$ and $\lambda_{2}^{\dagger}=2.5 \omega^{-1}$ to guarantee the emergence of a hysteresis region. To have an idea of the size of the correction terms $R_{a}$, we show the values of $R_{a}$ at equilibrium. The results of (1) $P L-2.3$, (2) $P L-3$, (3) PL-4, (4) Dolphins, (5) HIV, (6) Highschool, and (7) Jazz are shown in Figs. 3-9, respectively. In each figure, subfigures (a) and (b) correspond to the values of $\psi_{1}$, while (e) and (f) are the corresponding values of $R_{1}$. Similarly, (c) and (d) correspond to the values of $\psi_{2}$, while $(\mathrm{g})$ and $(\mathrm{h})$ are the corresponding values of $R_{2}$. We see that, for all the networks tested, the analytic phase diagram predicts quantitatively and accurately the outbreak transitions, while the predicted extinction transitions are qualitatively correct. The values of correction terms $R_{a}$ are near zero for the outbreak transitions, while have relatively larger magnitudes near extinction transitions.

\section{DISCUSSION}

We have analytically predicted the phase diagram of interacting SIS spreading dynamics using the technique of spectral dimension reduction and provided numerical validation. The analytic phase diagram elucidates the interplay

TABLE I. Basic topological features of seven real networks: $N$ and $M$ are the number of nodes and edges, respectively, $C$ is the clustering coefficient [44], $r$ is the assortative coefficient [45], $\langle k\rangle$ is the average degree, $H$ is the degree heterogeneity which defined as $H=\left\langle k^{2}\right\rangle /\langle k\rangle^{2}$, and $\langle d\rangle$ is the average shortest distance.

\begin{tabular}{lcccccccc}
\hline \hline & $N$ & $M$ & $C$ & $r$ & $k_{\max }$ & $\langle k\rangle$ & $H$ & $\langle d\rangle$ \\
\hline ER & 100 & 200 & 0.025 & 0.027 & 9 & 4 & 1.228 & 3.436 \\
$P L-2.3$ & 100 & 234 & 0.040 & -0.077 & 10 & 4.680 & 1.162 & 3.095 \\
$P L-3$ & 100 & 216 & 0.042 & -0.005 & 10 & 4.320 & 1.164 & 3.239 \\
$P L-4$ & 100 & 185 & 0.033 & 0.022 & 10 & 3.700 & 1.108 & 3.709 \\
Dolphins & 62 & 159 & 0.259 & -0.043 & 12 & 5.130 & 1.327 & 3.357 \\
HIV & 40 & 41 & 0.042 & -0.279 & 8 & 2.050 & 1.512 & 4.474 \\
Highschool & 70 & 274 & 0.465 & 0.083 & 19 & 7.829 & 1.190 & 2.640 \\
Jazz & 198 & 2742 & 0.618 & 0.021 & 100 & 27.697 & 1.396 & 2.236 \\
\hline \hline
\end{tabular}



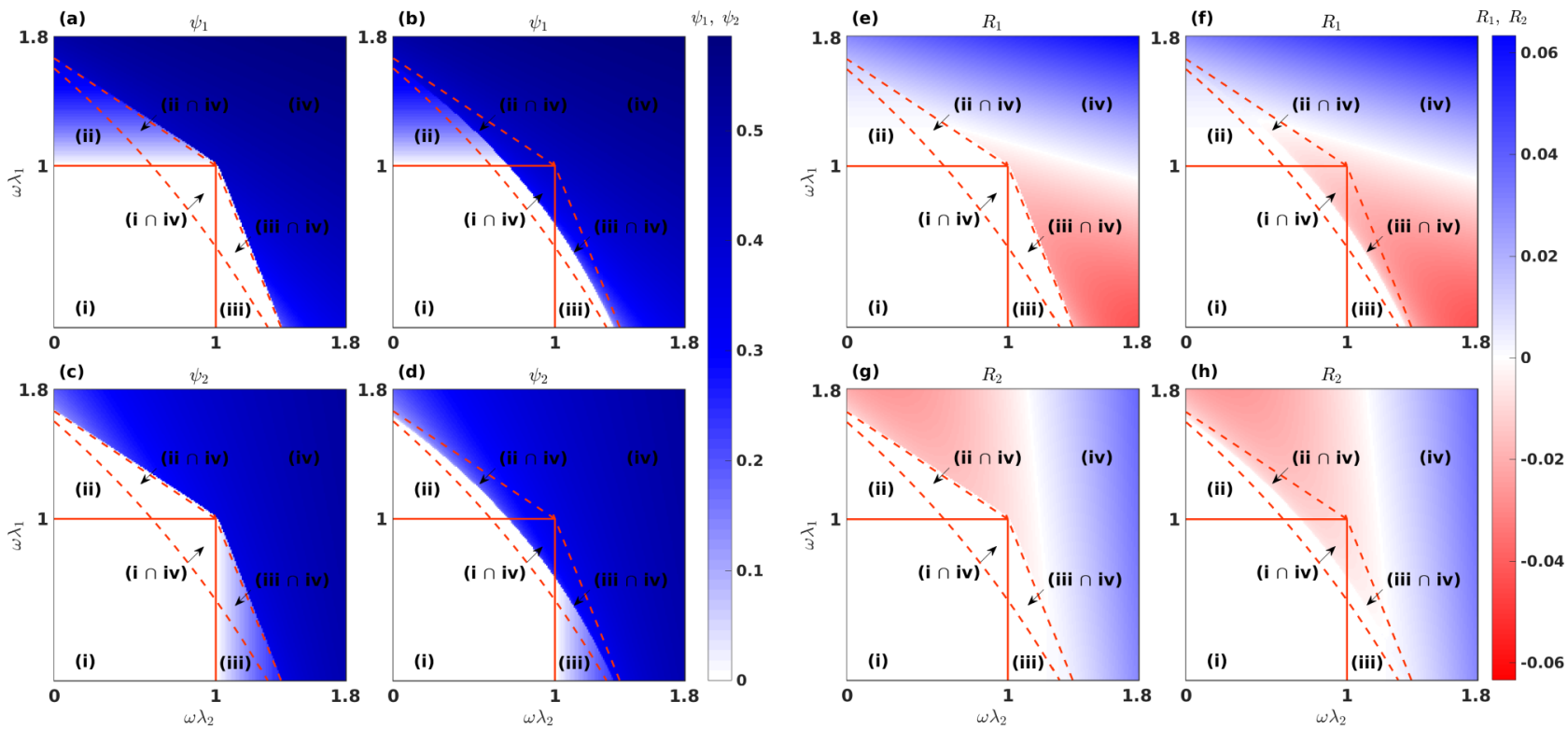

FIG. 3. Validation of analytic predictions for the power-law network with degree exponent $\beta=2.3$. The parameters of the spreading model are set as $\lambda_{1}^{\dagger}=3.5 \omega^{-1}$ and $\lambda_{2}^{\dagger}=2.5 \omega^{-1}$.

between discontinuous transitions and hystereses as well as the emergence of tricritical points. This method can also be applied to study other interacting epidemic models. For general epidemic models, a one-dimensional description of each epidemics is not sufficient [33]. Determining the number of macroscopic observables required for general epidemic models needs further exploration.

Previous theoretical methods for interacting spreading dynamics such as QMF theory [36] employ $2 N$ equations, where $N$ is the network size. For large networks, it is computationally demanding to solve the equations to determine the stability of the fixed points, as this requires manipulating the $2 N \times 2 N$ Jacobian matrix. It is thus infeasible to use the
QMF to map out the phase diagram for interacting spreading dynamics on complex networks, preventing us from gaining a full understanding of the interplay between network topology and the spreading dynamical process as a full phase diagram would reveal. The same difficulty arises with a naive application of the SDR method [33] to obtain the phase diagram for interacting spreading dynamics. In contrast, our approach gives a full picture of the phase diagram on large complex networks with an arbitrary topology through an effective twodimensional system, revealing rich phenomena that have not been systemically investigated. While many previous studies employed the mean-field theory to study different types of spreading dynamics on complex networks [46-48], our work
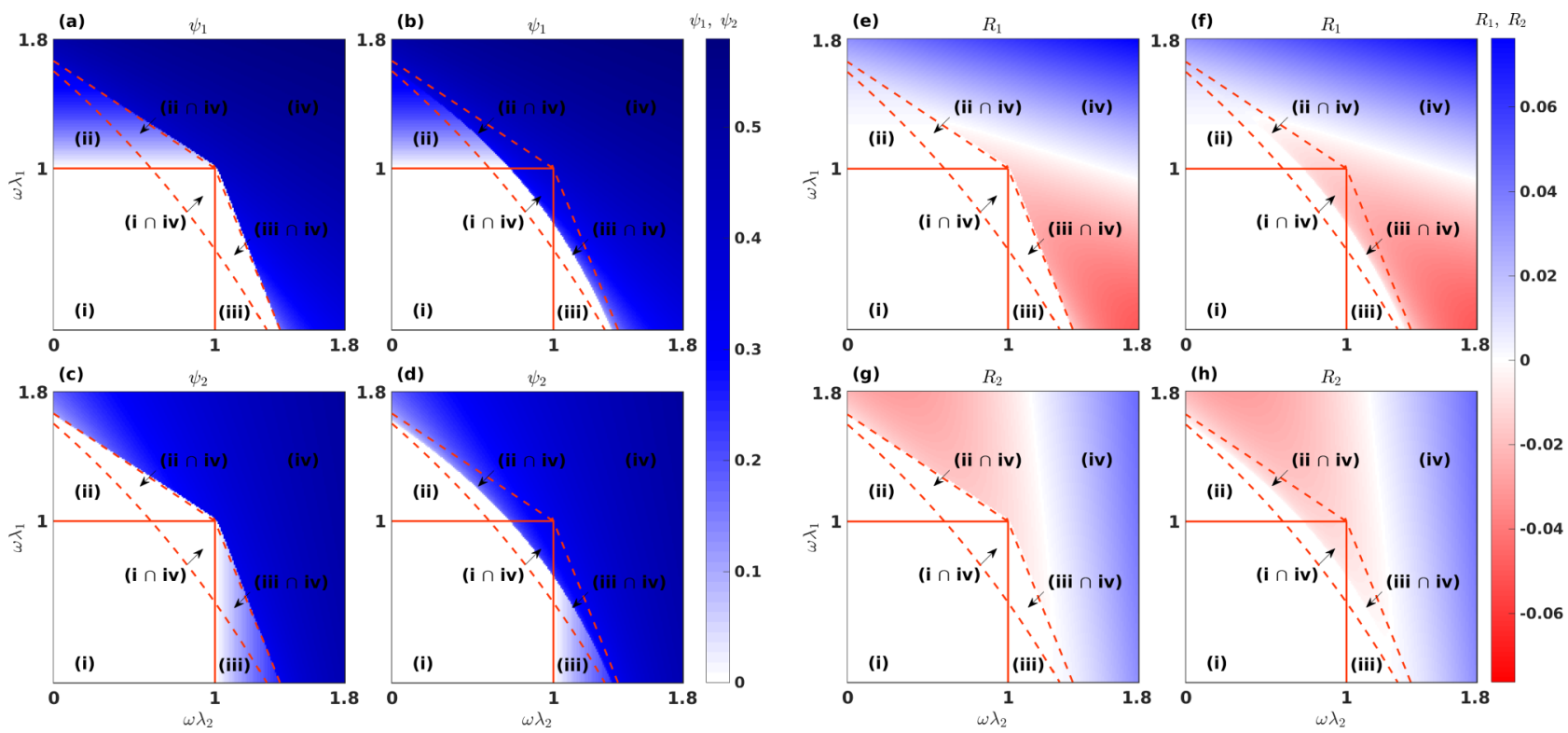

FIG. 4. Validation of analytic predictions for the power-law network with degree exponent $\beta=3$. The parameters of the spreading model are set as $\lambda_{1}^{\dagger}=3.5 \omega^{-1}$ and $\lambda_{2}^{\dagger}=2.5 \omega^{-1}$. 

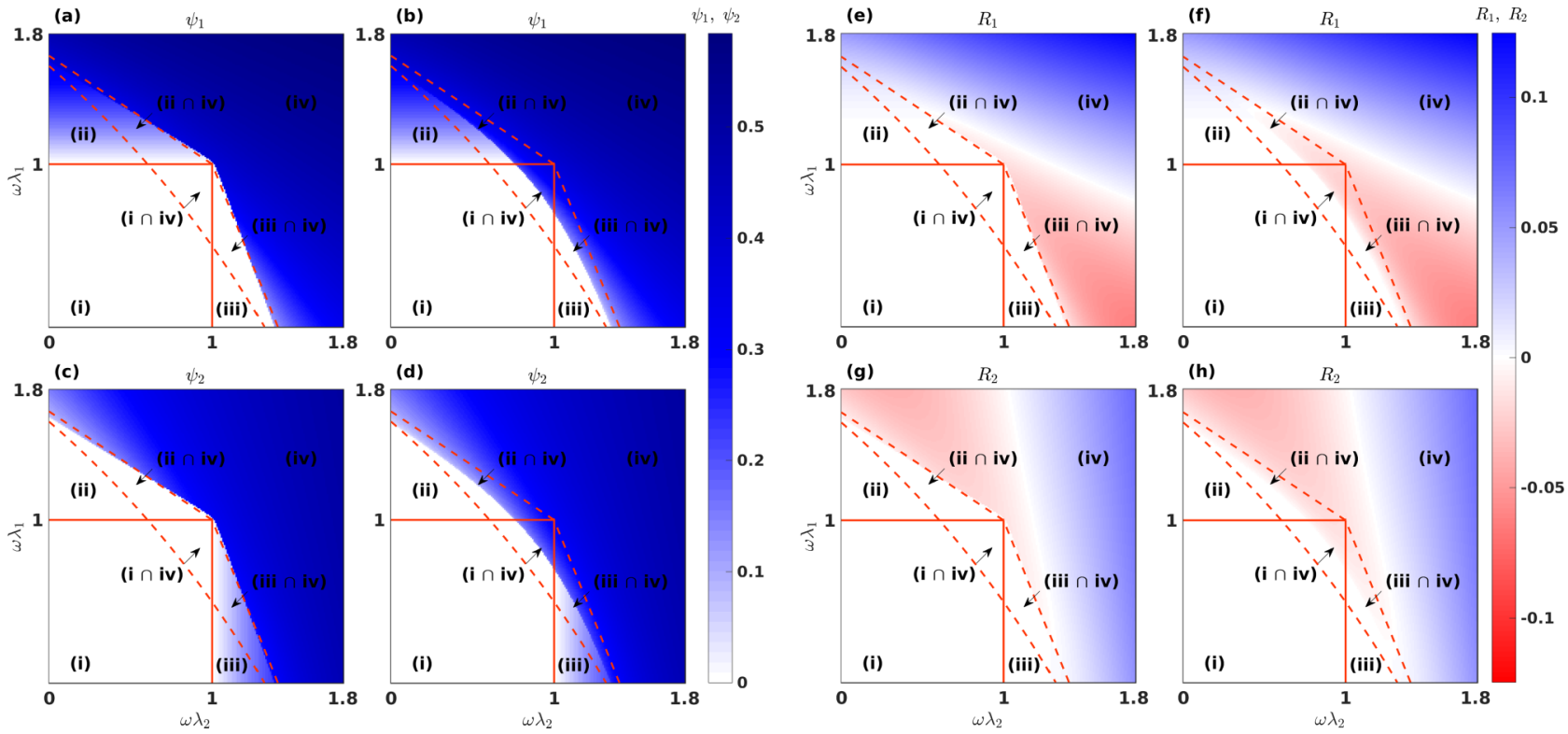

FIG. 5. Validation of analytic predictions for the power-law network with degree exponent $\beta=4$. The parameters of the spreading model are set as $\lambda_{1}^{\dagger}=3.5 \omega^{-1}$ and $\lambda_{2}^{\dagger}=2.5 \omega^{-1}$.

is not a simple application of the mean-field theory. In fact, we go way beyond by obtaining, for the first time to our knowledge, a global phase diagram laying out a clear picture of all possible dynamical states and the transitions among them through a comprehensive stability analysis-both at an unprecedented level of details.

Taken together, our work gives a full picture of the dependence of phase transition on network topology and spreading parameters for SIS dynamics, and thus lays a foundation for intervening or harnessing this type of interacting spreading processes. For instance, our phase diagram gives possible routes for controlling the type of phase transition through perturbations to the network structure or for controlling one spreading process through manipulating another interacting process. It should be cautioned that, while the SIS model provides phenomenological insights into relatively simply spreading processes and is thus a conceptually useful paradigmatic model, it may be too simplistic to describe spreading processes in the real world which can be significantly more complicated. To apply our analytic approach to irreversible epidemic processes beyond the SIS dynamics is possible but remains to be studied.
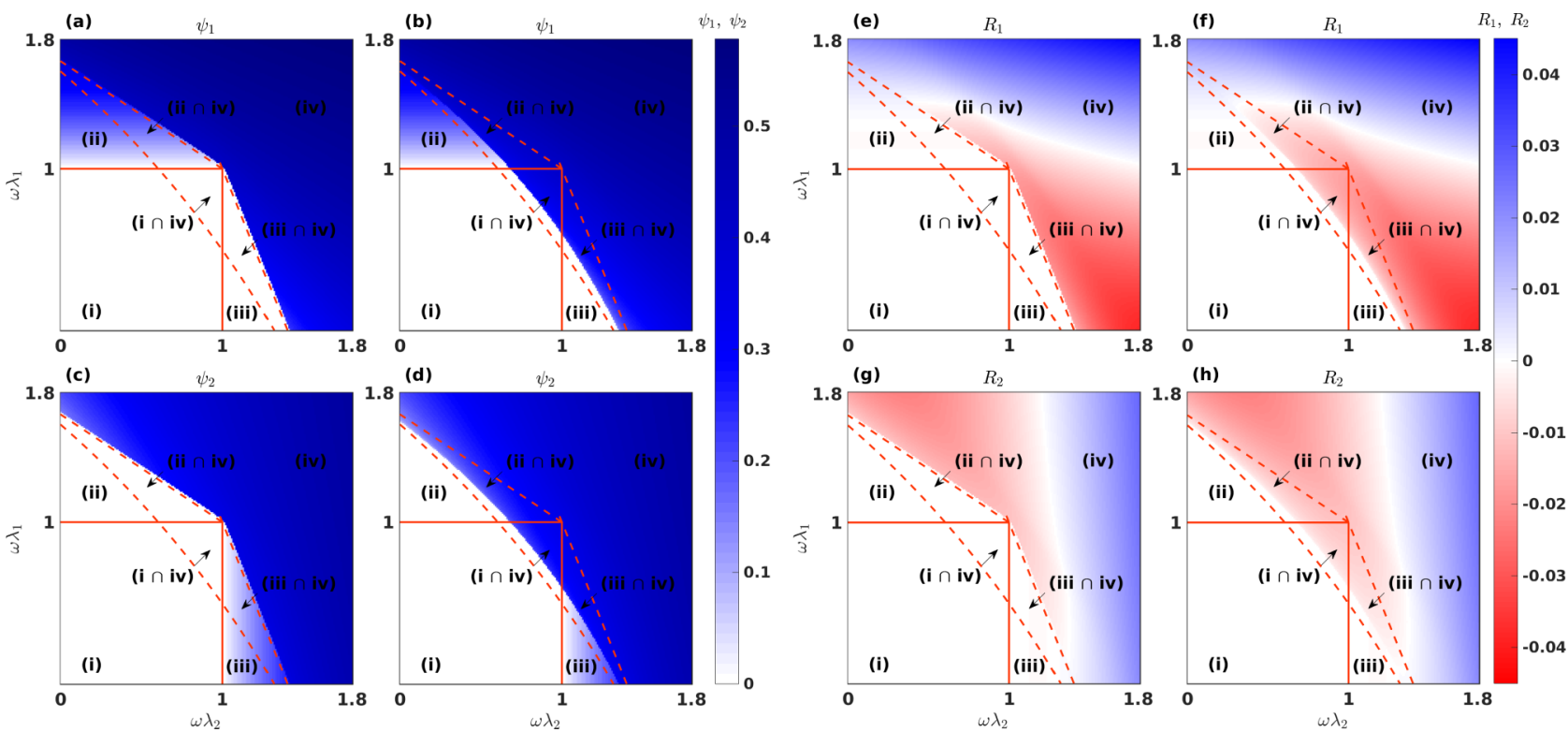

FIG. 6. Validation of analytic predictions for the Dolphins network. The parameters of the spreading model are set as $\lambda_{1}^{\dagger}=3.5 \omega^{-1}$ and $\lambda_{2}^{\dagger}=2.5 \omega^{-1}$. 

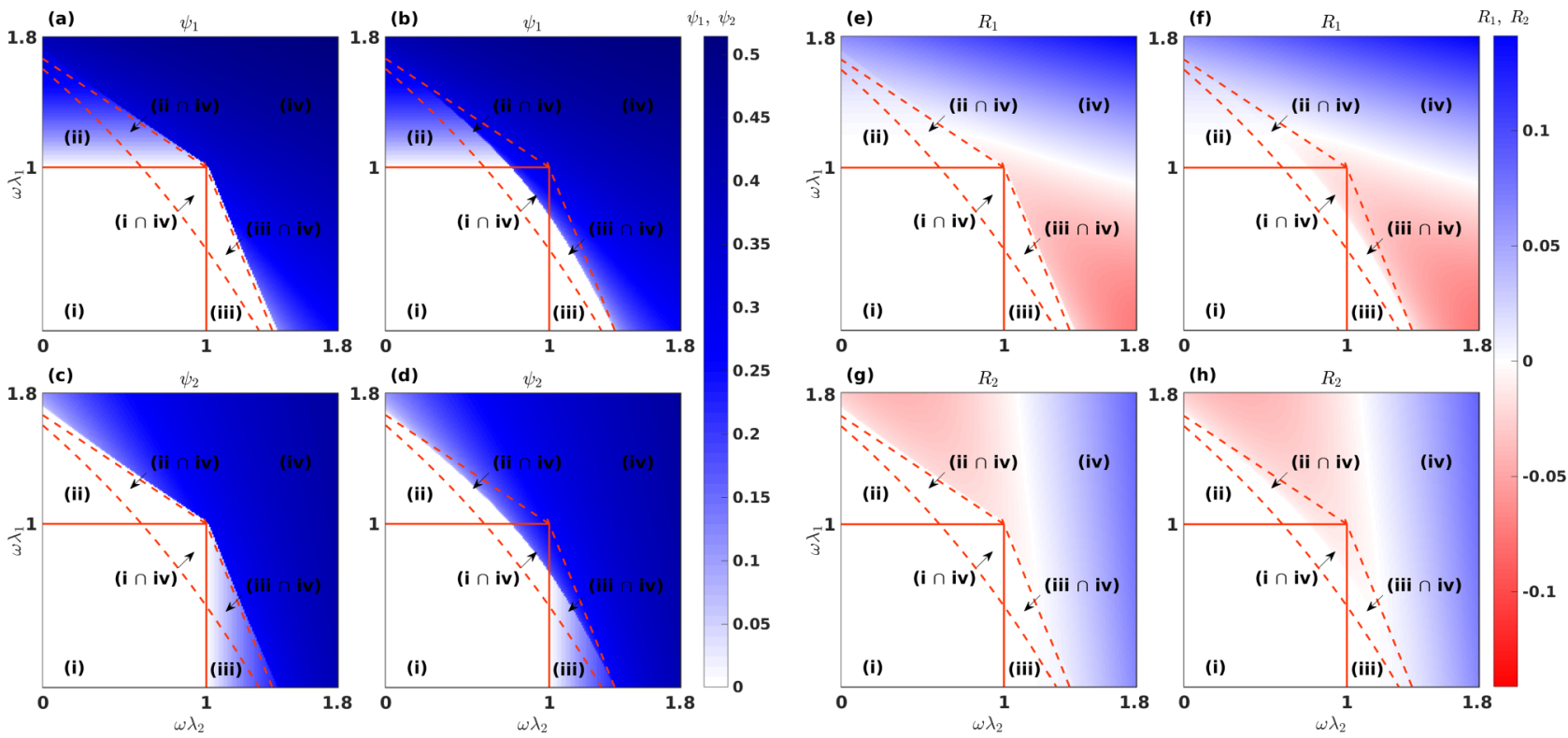

FIG. 7. Validation of analytic predictions for the HIV network. The parameters of the spreading model are set as $\lambda_{1}^{\dagger}=3.5 \omega^{-1}$ and $\lambda_{2}^{\dagger}=$ $2.5 \omega^{-1}$.

\section{ACKNOWLEDGMENTS}

This work was partially supported by NNSF of China under Grants No. 61903266, No. 61433014, and No. 61673086), China Postdoctoral Science Foundation under Grant No. 2018M631073, China Postdoctoral Science Special Foundation under Grant No. 2019T120829, Sichuan Science and Technology Program (No. 20YYJC4001), the Science Strength Promotion Program of the University of Electronic Science and Technology of China under Grant No. Y030190261010020, and Fundamental Research Funds for the Central Universities. Y.-C.L. is supported by ONR under Grant No. N00014-16-1-2828.

\section{APPENDIX: PROOF FOR $\mu \geqslant 1$}

To prove $\mu \geqslant 1$, we rewrite Eq. (10) as

$$
\mu^{-1}=\frac{\alpha^{T} G \alpha}{\alpha^{T} K \alpha} .
$$
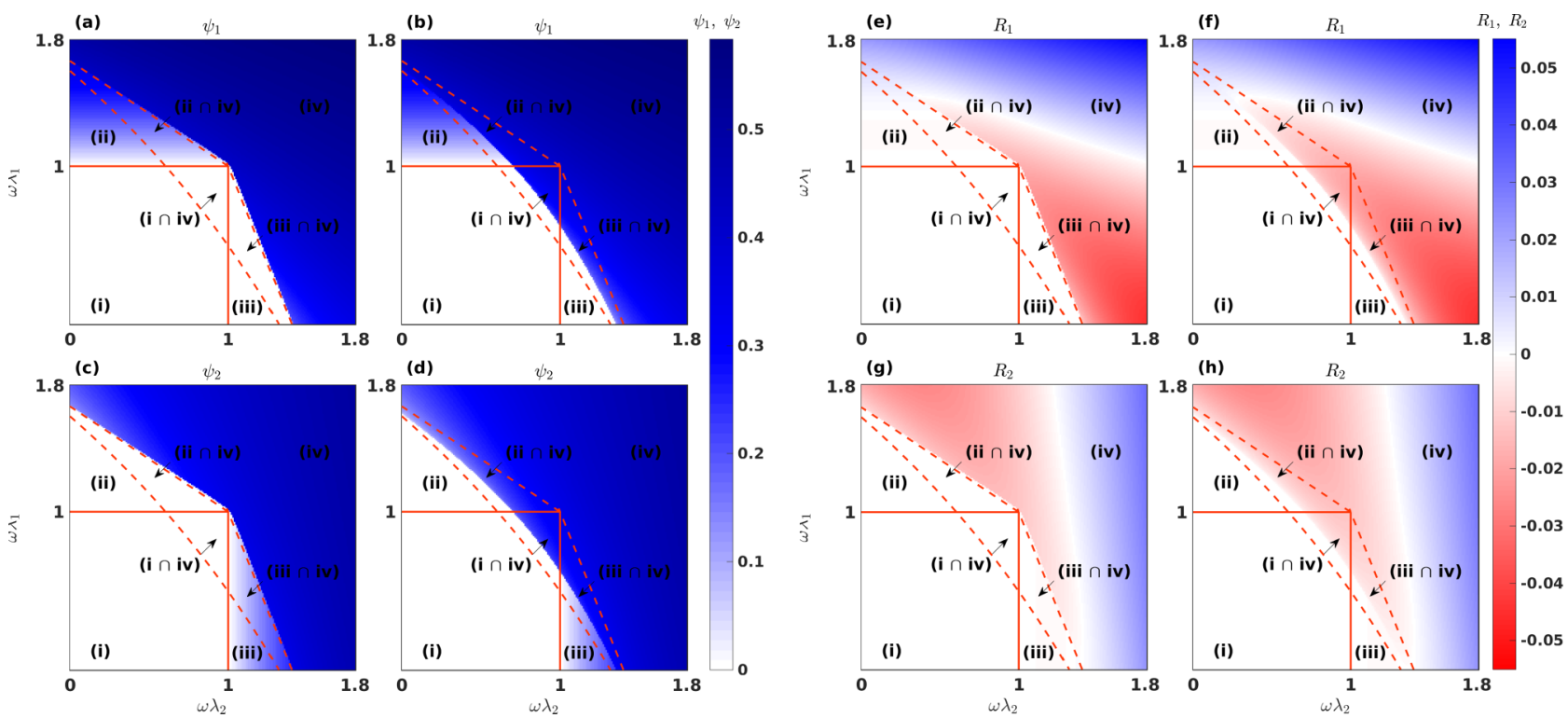

FIG. 8. Validation of analytic predictions for the Highschool network. The parameters of the spreading model are set as $\lambda_{1}^{\dagger}=3.5 \omega^{-1}$ and $\lambda_{2}^{\dagger}=2.5 \omega^{-1}$. 

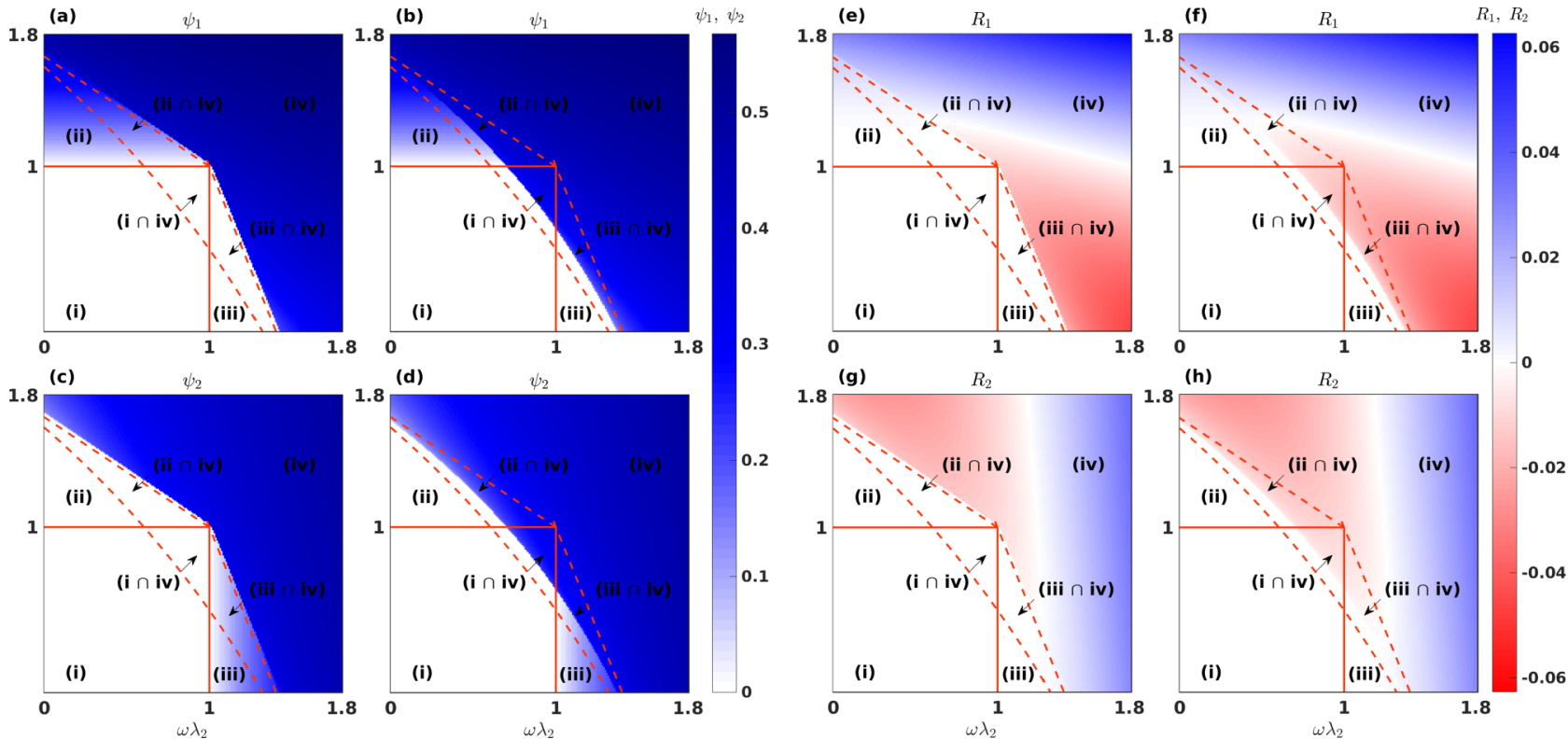

FIG. 9. Validation of analytic predictions for the Jazz network. The parameters of the spreading model are set as $\lambda_{1}^{\dagger}=3.5 \omega^{-1}$ and $\lambda_{2}^{\dagger}=$ $2.5 \omega^{-1}$.

Since $K$ is positive definite, it can be decomposed as $K=$ $K^{1 / 2} K^{1 / 2}$, where $K^{1 / 2}$ is a diagonal matrix whose entries are the square root of the degrees. Let $y=K^{1 / 2} \alpha$. We have $\alpha=$ $K^{-1 / 2} y$. Substituting this back to $\mu^{-1}$ gives

$$
\mu^{-1}=\frac{y K^{-1 / 2} G K^{-1 / 2} y}{y^{T} y},
$$

which is the Rayleigh quotient of matrix $K^{-1 / 2} G K^{-1 / 2}$ and, hence, we have $\mu^{-1} \leqslant \delta_{1}$, where $\delta_{1}$ is the largest eigenvalue of the matrix $K^{-1 / 2} G K^{-1 / 2}$. Recall that the symmetric normalized Laplacian matrix of $G$ is defined as

$$
L^{\mathrm{sym}}=I-K^{-1 / 2} G K^{-1 / 2},
$$

which has a smallest eigenvalue $\zeta_{n}=0$. As a result, we have $\delta_{1}=1-\zeta_{n}=1$, which gives $\mu \geqslant 1$.
[1] N. M. Ferguson, A. P. Galvani, and R. M. Bush, Ecological and immunological determinants of influenza evolution, Nature 422, 428 (2003).

[2] L. J. Abu-Raddad, P. Patnaik, and J. G. Kublin, Dual infection with HIV and malaria fuels the spread of both diseases in subSaharan Africa, Science 314, 1603 (2006).

[3] S. Funk, E. Gilad, C. Watkins, and V. A. Jansen, The spread of awareness and its impact on epidemic outbreaks, Proc. Nat. Acad. Sci. USA 106, 6872 (2009).

[4] J. R. Gog and B. T. Grenfell, Dynamics and selection of many-strain pathogens, Proc. Nat. Acad. Sci. USA 99, 17209 (2002).

[5] K. T. Eames and M. J. Keeling, Coexistence and specialization of pathogen strains on contact networks, Am. Nat. 168, 230 (2006).

[6] L. F. Berkman, I. Kawachi, and M. M. Glymour, Social Epidemiology (Oxford University Press, Oxford, 2014)

[7] J. Sanz, C.-Y. Xia, S. Meloni, and Y. Moreno, Dynamics of Interacting Diseases, Phys. Rev. X 4, 041005 (2014).

[8] R. Pastor-Satorras, C. Castellano, P. Van Mieghem, and A. Vespignani, Epidemic processes in complex networks, Rev. Mod. Phys. 87, 925 (2015).
[9] G. F. de Arruda, F. A. Rodrigues, and Y. Moreno, Fundamentals of spreading processes in single and multilayer complex networks, Phys. Rep. 756, 1 (2018).

[10] W. Wang, Q.-H. Liu, J. Liang, Y. Hu, and T. Zhou, Coevolution spreading in complex networks, Phys. Rep. 820, 1 (2019).

[11] D. Soriano-Paños, F. Ghanbarnejad, S. Meloni, and J. GómezGardeñes, Markovian approach to tackle the interaction of simultaneous diseases, Phys. Rev. E 100, 062308 (2019).

[12] M. M. Danziger, I. Bonamassa, S. Boccaletti, and S. Havlin, Dynamic interdependence and competition in multilayer networks, Nat. Phys. 15, 178 (2019).

[13] W. Cai, L. Chen, F. Ghanbarnejad, and P. Grassberger, Avalanche outbreaks emerging in cooperative contagions, Nat. Phys. 11, 936 (2015).

[14] J. D. Noh and H. Park, Asymmetrically Coupled Directed Percolation Systems, Phys. Rev. Lett. 94, 145702 (2005).

[15] C. Granell, S. Gómez, and A. Arenas, Dynamical Interplay Between Awareness and Epidemic Spreading in Multiplex Networks, Phys. Rev. Lett. 111, 128701 (2013).

[16] W. Wang, M. Tang, H. Yang, Y. Do, Y.-C. Lai, and G. Lee, Asymmetrically interacting spreading dynamics on complex layered networks, Sci. Rep. 4, 5097 (2014). 
[17] L. Hébert-Dufresne and B. M. Althouse, Complex dynamics of synergistic coinfections on realistically clustered networks, Proc. Nat. Acad. Sci. USA 112, 10551 (2015).

[18] L. Chen, Persistent spatial patterns of interacting contagions, Phys. Rev. E 99, 022308 (2019).

[19] C. Castellano, S. Fortunato, and V. Loreto, Statistical physics of social dynamics, Rev. Mod. Phys. 81, 591 (2009).

[20] S. N. Dorogovtsev, A. V. Goltsev, and J. F. F. Mendes, Critical phenomena in complex networks, Rev. Mod. Phys. 80, 1275 (2008).

[21] I. Z. Kiss, J. C. Miller, and P. L. Simon, Mathematics of Epidemics on Networks (Springer-Verlag, New York/Heidelberg/Dordrecht/London, 2017).

[22] L. J. Abu-Raddad, B. I. S. Van der Ventel, and N. M. Ferguson, Interactions of Multiple Strain Pathogen Diseases in the Presence of Coinfection, Cross Immunity, and Arbitrary Strain Diversity, Phys. Rev. Lett. 100, 168102 (2008).

[23] F. Zarei, S. Moghimi-Araghi, and F. Ghanbarnejad, Exact solution of generalized cooperative susceptible-infected-removed (SIR) dynamics, Phys. Rev. E 100, 012307 (2019).

[24] B. A. Prakash, A. Beutel, R. Rosenfeld, and C. Faloutsos, Winner takes all: Competing viruses or ideas on fair-play networks, in Proceedings of the 21st International Conference on World Wide Web, WWW'12 (ACM, New York, 2012), pp. 1037-1046.

[25] M. E. J. Newman, Threshold Effects for Two Pathogens Spreading on a Network, Phys. Rev. Lett. 95, 108701 (2005).

[26] L. Chen, F. Ghanbarnejad, W. Cai, and P. Grassberger, Outbreaks of coinfections: The critical role of cooperativity, Europhys. Lett. 104, 50001 (2013).

[27] F. Darabi Sahneh and C. Scoglio, Competitive epidemic spreading over arbitrary multilayer networks, Phys. Rev. E 89, 062817 (2014).

[28] C. Granell, S. Gómez, and A. Arenas, Competing spreading processes on multiplex networks: Awareness and epidemics, Phys. Rev. E 90, 012808 (2014).

[29] P. Van Mieghem, The N-intertwined SIS epidemic network model, Computing 93, 147 (2011).

[30] D. Achlioptas, R. M. D'Souza, and J. Spencer, Explosive percolation in random networks, Science 323, 1453 (2009).

[31] R. A. da Costa, S. N. Dorogovtsev, A. V. Goltsev, and J. F. F. Mendes, Explosive Percolation Transition is Actually Continuous, Phys. Rev. Lett. 105, 255701 (2010).

[32] J. Gao, B. Barzel, and A.-L. Barabási, Universal resilience patterns in complex networks, Nature 530, 307 (2016).
[33] E. Laurence, N. Doyon, L. J. Dubé, and P. Desrosiers, Spectral Dimension Reduction of Complex Dynamical Networks, Phys. Rev. X 9, 011042 (2019).

[34] J. Jiang, Z.-G. Huang, T. P. Seager, W. Lin, C. Grebogi, A. Hastings, and Y.-C. Lai, Predicting tipping points in mutualistic networks through dimension reduction, Proc. Nat. Acad. Sci. USA 115, E639 (2018).

[35] L. Chen, F. Ghanbarnejad, and D. Brockmann, Phase transitions and hysteresis of cooperative contagion processes, New J. Phys. 19, 103041 (2017).

[36] Y. Wang, D. Chakrabarti, C. Wang, and C. Faloutsos, Epidemic spreading in real networks: An eigenvalue viewpoint, in Proceedings of the 22nd International Symposium on Reliable Distributed Systems (IEEE, Piscataway, NJ, 2003), pp. 25-34.

[37] M. Boguná, C. Castellano, and R. Pastor-Satorras, Nature of the Epidemic Threshold for the Susceptible-Infected-Susceptible Dynamics in Networks, Phys. Rev. Lett. 111, 068701 (2013).

[38] B. A. Prakash, A. Beutel, R. Rosenfeld, and C. Faloutsos, Winner takes all: Competing viruses or ideas on fair-play networks, in Proceedings of the 21st International Conference on World Wide Web (ACM, New York, 2012), pp. 1037-1046.

[39] D. Lusseau, K. Schneider, O. J. Boisseau, P. Haase, E. Slooten, and S. M. Dawson, The bottlenose dolphin community of doubtful sound features a large proportion of long-lasting associations, Behav. Ecol. Sociobiol. 54, 396 (2003).

[40] D. M. Auerbach, W. W. Darrow, H. W. Jaffe, and J. W. Curran, Cluster of cases of the acquired immune deficiency syndrome: Patients linked by sexual contact, Am. J. Med. 76, 487 (1984).

[41] J. S. Coleman, Introduction to Mathematical Sociology (London Free Press, Glencoe, 1964).

[42] P. M. Gleiser and L. Danon, Community structure in jazz, Adv. Comp. Sys. 6, 565 (2003).

[43] J. Kunegis, Konect: the Koblenz network collection, in Proceedings of the 22nd International Conference on World Wide Web (ACM, New York, 2013), pp. 1343-1350.

[44] D. J. Watts and S. H. Strogatz, Collective dynamics of "smallworld" networks, Nature 393, 440 (1998).

[45] M. E. J. Newman, Assortative Mixing in Networks, Phys. Rev. Lett. 89, 208701 (2002).

[46] R. Pastor-Satorras and A. Vespignani, Epidemic Spreading in Scale-Free Networks, Phys. Rev. Lett. 86, 3200 (2001).

[47] M. Boguñá, R. Pastor-Satorras, and A. Vespignani, Absence of Epidemic Threshold in Scale-Free Networks with Degree Correlations, Phys. Rev. Lett. 90, 028701 (2003).

[48] C. Castellano and R. Pastor-Satorras, Non-Mean-Field Behavior of the Contact Process on Scale-Free Networks, Phys. Rev. Lett. 96, 038701 (2006). 